Amina Angelika Bouchentouf (Sidi Bel Abbes)

Abbes Rabhi (Sidi Bel Abbes)

Aboubacar Traore (Bamako)

\title{
CONSISTENCY OF RECURSIVE NONPARAMETRIC KERNEL ESTIMATES FOR INDEPENDENT FUNCTIONAL DATA
}

Abstract. We propose a new nonparametric estimator of the conditional hazard function. To this end we define nonparametric estimators of the conditional cumulative distribution and the density functions of a scalar response variable $Y$ given a functional random variable $X$. The conditional cumulative distribution, density and hazard functions for independent functional data are estimated nonparametrically. Our estimates are based on a recursive approach. We establish under appropriate conditions the almost sure and the quadratic average convergence rates of the resulting hazard rate estimator. Furthermore, a simulation study and an application to a real dataset illustrate our methodology.

1. Introduction. The estimation of the conditional hazard function (or hazard rate) is of great importance in statistics. The first fields of application of the hazard function were medicine and biology where the failure rate or interest rate risk have been studied. Currently, it is used in many fields such as medicine, geophysics, reliability, etc. for risk analysis, or the study of survival phenomena.

Historically, the recursive estimation with rate was introduced in [WW69. Later, BL94] proposed a simple recursive estimation method for linear regression models with $\mathrm{AR}(p)$ disturbances. A recent application of recursive

2010 Mathematics Subject Classification: 62G05, 62G99, 62M10.

Key words and phrases: conditional density function, conditional cumulative distribution function, conditional hazard function, recursive estimation, functional random variable, kernel estimator, semi-metric space.

Received 17 May 2018; revised 21 July 2018 and 11 September 2018.

Published online 22 March 2019. 
methods has been given in [AT16], where regression estimation was studied by local polynomial fitting for multivariate data streams.

The objective of our work is to propose a nonparametric family of recursive kernel estimators of the conditional hazard function by adapting to the functional case the result given in [R92].

Most of the existing works in the conditional case treat only the case where the explanatory variable is a real or a vector. The literature in the case where the data is of a functional nature (a curve) is very limited. The first result in this context was given by [FRV08], establishing the almost complete convergence of the conditional hazard function estimator in the independent case. For more information on functional data analysis, see [C14, GV16, ABCV17] for recent advances and [GKWW18] for some selected statistical methods of data analysis for multivariate functional data.

By definition, the conditional hazard function depends on both the conditional density and the conditional survival functions. The first uniform results on the estimation of the distribution function conditioned on a functional variable were established in the thesis [T11]. From the recent literature, we can cite [LM14] establishing the quadratic mean convergence and the asymptotic normality of the estimator of the conditional hazard function, considering a strictly stationary random field (process) $Z_{i}=\left(X_{i}, Y_{i}\right)_{i \in \mathbb{N}^{\mathbb{N}}}$, when the univariate response variable $Y_{i}$ is conditioned on the functional explanatory variable $X_{i}$. The asymptotic normality of the kernel estimator of the conditional distribution function was studied in [B14], where a new nonparametric estimator of the conditional cumulative distribution of a scalar response variable $Y$ given a functional random variable $X$ was introduced. This estimate was based on a recursive approach. Under certain conditions, the asymptotic normality of the model was proved.

Nonparametric kernel estimates (conditional density and conditional cumulative functions) are used in many fields. For instance, in finance (see [S04, C08]) they are used in the estimation of the CVAR (Conditional Valueat-Risk) and the CES (Conditional Expected Shortfall). The VAR [EKM10] is currently one of the most popular risk measures employed in finance; it measures the financial risk associated with losses and is determined for a given probability level $\alpha$, while the Expected Shortfall (ES) A02 is the average of the $100(1-\alpha) \%$ bad losses.

The aim of this paper is to consider a recursive estimation of the conditional hazard function, exhibiting a clear advantage as regards computation time compared to the classical (nonrecursive) estimator. To this end the estimations of the conditional cumulative distribution and conditional density functions of the scalar response given functional random variables are established using the recursive estimation method. So, we are interested in nonparametric estimation based on conditional recursive kernels as well as its 
applications to prediction. The main objective is to improve the performance of nonparametric conditional kernel predictors, reducing their computation time by using recursive kernels. The recursive property is obviously useful in sequential surveys and for large samples, because addition of a new observation means that nonrecursive estimators need to be recalculated. In addition, we need to store complete data to calculate them. Consequently, the simulation time is reduced compared to the classical estimation.

Moreover, note that a direct application of nonparametric estimation is the estimation of conditional laws. Both functional conditional distributions and density functions are used in econometrics [F11]. These conditional models are used for prediction of time series through conditional quantiles and mode.

The outline of this paper is as follows: In Section 2 we define the model and the estimates; the recursive estimators of the conditional cumulative distribution, conditional density and conditional hazard functions. In Section 3 we make some hypotheses useful for our study. The almost sure and the quadratic mean convergence rates of the proposed estimators are given in Section 4. Section 5 analyzes the performance of our estimator using a simulation study and a real dataset. In Section 6 the proofs of the main results of Section 4 are given. Finally, in Section 7 we give some concluding remarks.

2. Model and notations. Let $\left(X_{1}, Y_{1}\right), \ldots,\left(X_{n}, Y_{n}\right)$ be a sample of independent pairs identically distributed as $(X, Y)$ which is a random pair valued in $\mathcal{F} \times \mathbb{R}$, where $(\mathcal{F}, d(;))$ is a semi-metric space.

Let

$$
h^{[x]}(y)=\frac{f^{[x]}(y)}{1-F^{[x]}(y)}, \quad \forall y \in \mathbb{R}, \quad \text { when } \quad F^{[x]}(y)<1,
$$

be the conditional hazard function, where $f^{[x]}(y)$ and $F^{[x]}(y)$ are respectively the conditional density and the distribution functions [BRS15].

Define

$$
\widehat{h}_{n}^{[x, l]}(y)=\frac{\widehat{f}_{n}^{[x, l]}(y)}{1-\widehat{F}_{n}^{[x, l]}(y)},
$$

as the conditional hazard function estimator, where $\widehat{f}_{n}^{[x, l]}(y)$ and $\widehat{F}_{n}^{[x, l]}(y)$ are respectively the conditional density and distribution functions estimators.

The conditional distribution function estimator is defined by

$$
\widehat{F}_{n}^{[x, l]}(y)=\frac{\sum_{i=1}^{n} \frac{1}{\left[F\left(h_{i}\right)\right]^{l}} K\left(\frac{\left\|x-X_{i}\right\|}{h_{i}}\right) H\left(\frac{y-Y_{i}}{h_{i}}\right)}{\sum_{i=1}^{n} \frac{1}{\left[F\left(h_{i}\right)\right]^{l}} K\left(\frac{\left\|x-X_{i}\right\|}{h_{i}}\right)},
$$

where $K$ is a kernel, $H$ a distribution function, $h_{n}$ a sequence of positive 
reals, $l$ a parameter belonging to $[0,1], d\left(x, X_{i}\right)=\left\|x-X_{i}\right\|$ and $F\left(h_{i}\right)=$ $\mathbb{P}\left(\left\|x-X_{i}\right\| \leq h_{i}\right)$. Our family of estimators is a recursive modification of the estimate just defined and can be computed recursively by

$$
\widehat{F}_{n+1}^{[x, l]}(y)=\frac{\left(\sum_{i=1}^{n}\left(F\left(h_{i}\right)\right)^{1-l}\right) \varphi_{n}^{[x, l]}(y)}{\left(\sum_{i=1}^{n}\left(F\left(h_{i}\right)\right)^{1-l}\right) f_{n}^{[l]}(x)+\left(\sum_{i=1}^{n+1}\left(F\left(h_{i}\right)\right)^{1-l}\right) K_{n+1}^{[l]}\left(\left\|x-X_{n+1}\right\|\right)},
$$

where

$$
\begin{aligned}
\varphi_{n}^{[x, l]}(y) & =\frac{\sum_{i=1}^{n} \frac{H\left(\frac{y-Y_{i}}{h_{i}}\right)}{\left[F\left(h_{i}\right)\right]^{l}} K\left(\frac{\left\|x-X_{i}\right\|}{h_{i}}\right)}{\sum_{i=1}^{n}\left[F\left(h_{i}\right)\right]^{1-l}}, \\
f_{n}^{[l]}(x) & =\frac{\sum_{i=1}^{n} \frac{1}{\left[F\left(h_{i}\right)\right]^{l}} K\left(\frac{\left\|x-X_{i}\right\|}{h_{i}}\right)}{\sum_{i=1}^{n}\left[F\left(h_{i}\right)\right]^{1-l}}, \\
K_{i}^{[l]}(\cdot) & =\frac{1}{\left[F\left(h_{i}\right)\right]^{l} \sum_{j=1}^{i}\left[F\left(h_{j}\right)\right]^{1-l}} K\left(\frac{\cdot}{h_{i}}\right) .
\end{aligned}
$$

Next, the conditional density estimator is defined by

$$
\widehat{f}_{n}^{[x, l]}(y)=\frac{\sum_{i=1}^{n} \frac{1}{h_{i}\left[F\left(h_{i}\right)\right]^{l}} K\left(\frac{\left\|x-X_{i}\right\|}{h_{i}}\right) H^{\prime}\left(\frac{y-Y_{i}}{h_{i}}\right)}{\sum_{i=1}^{n} \frac{1}{\left[F\left(h_{i}\right)\right]^{]}} K\left(\frac{\left\|x-X_{i}\right\|}{h_{i}}\right)} .
$$

This estimator can also be computed recursively as

$$
\widehat{f}_{n+1}^{[x, l]}(y)=\frac{\left(\sum_{i=1}^{n}\left(F\left(h_{i}\right)\right)^{1-l}\right) \phi_{n}^{[x, l]}(y)}{\left(h_{n+1}\right)^{-1}\left(\sum_{i=1}^{n+1}\left(F\left(h_{i}\right)\right)^{1-l}\right) H^{\prime}\left(\frac{y-Y_{n+1}}{h_{n+1}}\right) K_{n+1}^{[l]}\left(\left\|x-X_{n+1}\right\|\right)},
$$

where

$$
\phi_{n}^{[x, l]}(y)=\frac{\sum_{i=1}^{n} \frac{1}{h_{i}\left[F\left(h_{i}\right)\right]^{l}} K\left(\frac{\left\|x-X_{i}\right\|}{h_{i}}\right) H^{\prime}\left(\frac{y-Y_{i}}{h_{i}}\right)}{\sum_{i=1}^{n}\left[F\left(h_{i}\right)\right]^{1-l}} .
$$

Finally, the conditional hazard function estimator is

$$
\widehat{h}_{n}^{[x, l]}(y)=\frac{\sum_{i=1}^{n} \frac{1}{h_{i}\left[F\left(h_{i}\right)\right]^{l}} K\left(\frac{\left\|x-X_{i}\right\|}{h_{i}}\right) H^{\prime}\left(\frac{y-Y_{i}}{h_{i}}\right)}{\sum_{i=1}^{n} \frac{1}{\left[F\left(h_{i}\right)\right]^{l}} K\left(\frac{\left\|x-X_{i}\right\|}{h_{i}}\right)\left[1-H\left(\frac{y-Y_{i}}{h_{i}}\right)\right]} .
$$

This estimator is determined recursively as

$$
\begin{gathered}
\widehat{h}_{n+1}^{[x, l]}(y)=\frac{\left[\sum_{i=1}^{n}\left(F\left(h_{i}\right)\right)^{1-l}\right] \phi_{n}^{[x, l]}(y)}{+\left(h_{n+1}\right)^{-1}\left[\sum_{i=1}^{n+1}\left(F\left(h_{i}\right)\right)^{1-l}\right] H^{\prime}\left(\frac{y-Y_{n+1}}{h_{n+1}}\right) K_{n+1}^{[l]}\left(\left\|x-X_{n+1}\right\|\right)} \\
\quad \begin{array}{c}
{\left[\sum_{i=1}^{n}\left(F\left(h_{i}\right)\right)^{1-l}\right] \tilde{\varphi}_{n}^{[x, l]}(y)} \\
\quad+\left[\sum_{i=1}^{n+1}\left(F\left(h_{i}\right)\right)^{1-l}\right] K_{n+1}^{[l]}\left(\left\|x-X_{n+1}\right\|\right)\left[1-H\left(\frac{y-Y_{n+1}}{h_{n+1}}\right)\right]
\end{array}
\end{gathered}
$$


where

$$
\tilde{\varphi}_{n}^{[x, l]}(y)=\frac{\sum_{i=1}^{n} \frac{1}{\left[F\left(h_{i}\right)\right]^{l}} K\left(\frac{\left\|x-X_{i}\right\|}{h_{i}}\right)\left[1-H\left(\frac{y-Y_{i}}{h_{i}}\right)\right]}{\sum_{i=1}^{n}\left[F\left(h_{i}\right)\right]^{1-l}} .
$$

The following notations from [FMV07] will be needed:

$$
\begin{aligned}
& M_{0}=K(1)-\int_{0}^{1}(s K(s))^{\prime} \tau_{0}(s) d s, \\
& M_{1}=K(1)-\int_{0}^{1} K^{\prime}(s) \tau_{0}(s) d s, \\
& M_{2}=K^{2}(1)-\int_{0}^{1}\left(K^{2}(s)\right)^{\prime} \tau_{0}(s) d s, \quad \text { where } \tau_{0}(s)=\lim _{h \rightarrow 0} \frac{F(h s)}{F(h)}<\infty .
\end{aligned}
$$

3. Assumptions. In order to establish the recursive estimation of the conditional hazard function we assume that the following hypotheses hold:

(H1) $K$ is a bounded kernel with compact support $[0,1]$ such that there exist constants $c_{1}$ and $c_{2}$ with $0<c_{1}<K(t)<c_{2}<\infty$.

(H2) (i) The sequence $\left\{h_{i}, i \geq 1\right\}$ of bandwidths satisfies $0<h_{i} \downarrow 0$ as $i \rightarrow \infty$.

(ii) If $h_{n} \rightarrow 0$ then $F\left(h_{n}\right) \rightarrow F(0)=0$ as $n \rightarrow \infty$, and $\forall s \in[0,1]$, $\tau_{h}(s)=\frac{F(h s)}{F(h)} \rightarrow \tau_{0}(s)<\infty$ as $h \rightarrow 0$.

(H3) If $\lim _{n \rightarrow \infty} n F\left(h_{n}\right)=\infty$ then, as $n \rightarrow \infty$ :

(i) $A_{n, l}:=\frac{1}{n} \sum_{i=1}^{n} \frac{h_{i}}{h_{n}}\left[\frac{F\left(h_{i}\right)}{F\left(h_{n}\right)}\right]^{1-l} \rightarrow \alpha_{[l]}<\infty$.

(ii) $\forall r \leq 2, B_{n, r}:=\frac{1}{n} \sum_{i=1}^{n}\left[\frac{F\left(h_{i}\right)}{F\left(h_{n}\right)}\right]^{r} \rightarrow \beta_{[r]}<\infty$.

(iii) $P:=\frac{1}{n} \sum_{i=1}^{n} \frac{1}{h_{i}}\left[\frac{F\left(h_{i}\right)}{F\left(h_{n}\right)}\right]^{1-2 l} \rightarrow \varpi_{[1-2 l]}<\infty$.

(H4) There exists $\mu>0$ such that

$$
\lim _{n \rightarrow \infty} \frac{n F\left(h_{n}\right)(\ln n)^{-1-2 / \mu}}{(\ln \ln n)^{2(\alpha+1)}}=\infty \quad \text { and } \quad \lim _{n \rightarrow \infty}(\ln n)^{2 / \mu} F\left(h_{n}\right)=0
$$

for some $\alpha \geq 0$.

(H5) (i) $H$ is a $C^{2}$ function with compact support.

(ii) $\exists b_{1}, b_{2}>2, \forall y_{1}, y_{2} \in \mathbb{R}, \forall\left(x_{1}, x_{2}\right) \in N_{x}^{2}$,

$$
\left|F^{\left[x_{1}\right]}\left(y_{1}\right)-F^{\left[x_{2}\right]}\left(y_{2}\right)\right| \leq\left(d\left(x_{1}, x_{2}\right)^{b_{1}}+\left|y_{1}-y_{2}\right|^{b_{2}}\right) \text {. }
$$


(iii) $\forall y_{1}, y_{2} \in \mathbb{R}, \forall\left(x_{1}, x_{2}\right) \in N_{x}^{2}$,

$$
\left|f^{\left[x_{1}\right]}\left(y_{1}\right)-f^{\left[x_{2}\right]}\left(y_{2}\right)\right| \leq\left(d\left(x_{1}, x_{2}\right)^{b_{1}}+\left|y_{1}-y_{2}\right|^{b_{2}}\right),
$$

with $d\left(x_{1}, x_{2}\right)=\left\|x_{1}-x_{2}\right\|$ and $N_{x}^{2}$ a fixed neighborhood of $x$.

(H6)

(i) $C_{n, l}:=\frac{1}{n} \sum_{i=1}^{n} h_{i}^{b} \frac{\left(F\left(h_{i}\right)\right)^{a}}{\left(F\left(h_{n}\right)\right)^{1-2 l}} \rightarrow 0$ as $n \rightarrow \infty$ for any $b \geq$ $\max \left(b_{1}-2 ; b_{2}-2\right)$ and any $a \leq 2$.

(ii) The function $\varphi$ is differentiable at 0 , where

$$
\begin{aligned}
\varphi(t) & =E\left\{\left[H\left(\frac{y-Y_{i}}{h_{i}}\right)-F^{[x]}(y)\right] \mid\left\|x-X_{i}\right\|=t\right\} \\
& =E\left\{\left[\int_{\mathbb{R}} H^{\prime}(t) F^{[X]}\left(y-h_{i} t\right) d t-F^{[x]}(y)\right] \mid\left\|x-X_{i}\right\|=t\right\} .
\end{aligned}
$$

(iii) The function $\phi$ is differentiable at 0 , where

$$
\begin{aligned}
\phi(t) & =E\left\{\left[\frac{1}{h_{i}} H^{\prime}\left(\frac{y-Y_{i}}{h_{i}}\right)-f^{[x]}(y)\right] \mid\left\|x-X_{i}\right\|=t\right\} \\
& =E\left\{\left[\int_{\mathbb{R}} H^{\prime}(t) f^{[X]}\left(y-h_{i} t\right) d t-f^{[x]}(y)\right] \mid\left\|x-X_{i}\right\|=t\right\} .
\end{aligned}
$$

(H7) $\exists v<\infty, \forall(t, y) \in N_{x} \times \mathbb{R}, f^{[x]}(y) \leq v$.

(H8) $\exists \beta<\infty, \forall(t, y) \in N_{x} \times \mathbb{R}, F^{[x]}(y) \leq 1-\beta$.

Remark 3.1. By using (H1) and (H2) we can prove that $M_{0}, M_{1}$ and $M_{2}$ are finite. By using (H5)(i-iii), we have

$$
\begin{aligned}
\sigma_{\varepsilon i}^{2}(X) & =\operatorname{Var}\left[H\left(\frac{y-Y_{i}}{h_{i}}\right) \mid X\right]=o\left(h_{i}^{\left(b_{2}-1\right)}\right)+F^{[x]}(y)\left(1-F^{[x]}(y)\right) \\
& \underset{i \rightarrow \infty}{\longrightarrow} \sigma_{\varepsilon}^{2}(X)=F^{[x]}(y)\left(1-F^{[x]}(y)\right)
\end{aligned}
$$

and

$$
\begin{aligned}
h_{i} \theta_{\varepsilon i}^{2}(X) & =h_{i} \operatorname{Var}\left[\frac{1}{h_{i}} H^{\prime}\left(\frac{y-Y_{i}}{h_{i}}\right) \mid X\right] \\
& \underset{i \rightarrow \infty}{\longrightarrow} \theta_{\varepsilon}^{2}(X)=f^{[x]}(y) \int_{\mathbb{R}} H^{\prime 2}(t) d t .
\end{aligned}
$$

4. Main results. As the conditional hazard function depends on the conditional density and the conditional distribution functions, its asymptotic properties depend naturally on those of the two functions. First, let us start with the asymptotic properties of the conditional distribution function. The almost sure convergence is presented in the following theorem. 
Theorem 4.1. Suppose that (H1)-(H4), (H5)(i-ii) and (H6)(i-iii) are satisfied. If $\lim _{n \rightarrow \infty} n h_{n}^{2}=0$, then

$$
\begin{aligned}
\limsup _{n \rightarrow \infty}\left[\frac{n F\left(h_{n}\right)}{\ln \ln n}\right]^{1 / 2}\left[\widehat{F}_{n}^{[x, l]}(y)-F^{[x]}(y)\right] & \\
= & \frac{\left[2 M_{2} \beta_{[1-2 l]} F^{[x]}(y)\left(1-F^{[x]}(y)\right)\right]^{1 / 2}}{M_{1} \beta_{[1-l]}} \quad \text { a.s. }
\end{aligned}
$$

REMARK 4.2. Bandwidths and small ball probabilities are typically chosen such that the condition $\lim _{n \rightarrow \infty} n h_{n}^{2}=0$ is satisfied; for more details see [ACT14].

The next Theorem concerns the quadratic mean convergence of the conditional distribution function.

Theorem 4.3. Assume that (H1)-(H3), (H5)(i-ii) and (H6)(i-iii) are satisfied. If there is a constant $c>0$ such that $n F\left(h_{n}\right) h_{n}^{2} \rightarrow c$ as $n \rightarrow \infty$, then

$$
\begin{aligned}
\lim _{n \rightarrow \infty} n F\left(h_{n}\right) E\left[\widehat{F}_{n}^{[x, l]}(y)-F^{[x]}(y)\right]^{2} \\
=\frac{\beta_{[1-2 l]}}{\beta_{[1-l]}^{2}} \frac{M_{2}}{M_{1}^{2}} F^{[x]}(y)\left(1-F^{[x]}(y)\right)+c\left[\varphi^{\prime}(0)\right]^{2} \frac{\alpha_{[l]}^{2}}{\beta_{[1-l]}^{2}} \frac{M_{0}^{2}}{M_{1}^{2}} .
\end{aligned}
$$

The quadratic mean convergence of the conditional distribution function depends on the asymptotic bias and variance of the estimator, thus we introduce the following lemmas.

Lemma 4.4. Suppose that (H1)-(H3), (H5)(i-ii) and (H6)(i-iii) are satisfied. If there is a constant $c>0$ such that $n F\left(h_{n}\right) h_{n}^{2} \rightarrow c$ as $n \rightarrow \infty$, then

$$
E\left[\widehat{F}_{n}^{[x, l]}(y)-F^{[x]}(y)\right]=h_{n} \varphi^{\prime}(0) \frac{\alpha_{[l]}}{\beta_{[1-l]}} \frac{M_{0}}{M_{1}}[1+o(1)]+O\left[\frac{1}{n F\left(h_{n}\right)}\right] .
$$

The asymptotic bias of our estimator $\left(E\left[\widehat{F}_{n}^{[x, l]}(y)-F^{[x]}(y)\right] \simeq O\left[\frac{1}{n F\left(h_{n}\right)}\right]\right)$ is equivalent to that of the classical one for independent [BRS15] and dependent [D08] cases.

Lemma 4.5. Suppose that (H1)-(H3), (H5)(i-ii) and (H6)(i-iii) are satisfied. If there is a constant $c>0$ such that $n F\left(h_{n}\right) h_{n}^{2} \rightarrow c$ as $n \rightarrow \infty$, then

$$
\lim _{n \rightarrow \infty} n F\left(h_{n}\right) \operatorname{Var}\left[\widehat{F}_{n}^{[x, l]}(y)\right]=\frac{\beta_{[1-2 l]}}{\beta_{[1-l]}^{2}} \frac{M_{2}}{M_{1}^{2}} \sigma_{\varepsilon}^{2}(X) .
$$

In practice, if we take $c=0$ in Theorem 4.3 we get the following corollary. 
Corollary 4.6. Suppose that (H1)-(H3), (H5)(i-ii) and (H6)(i-iii) are satisfied. If $n F\left(h_{n}\right) h_{n}^{2} \rightarrow 0$ as $n \rightarrow \infty$, then

$$
\lim _{n \rightarrow \infty} n F\left(h_{n}\right) E\left[\widehat{F}_{n}^{[x, l]}(y)-F^{[x]}(y)\right]^{2}=\frac{\beta_{[1-2 l]}}{\beta_{[1-l]}^{2}} \frac{M_{2}}{M_{1}^{2}} F^{[x]}(y)\left(1-F^{[x]}(y)\right) .
$$

The asymptotic variance of our conditional cumulative estimate is equivalent to that of the nonrecursive estimate for independent data BRS15, and smaller than in the case where the data are dependent [D08], given as $O\left(\frac{(\ln n)^{2}}{n F\left(h_{n}\right)}\right)$.

Next, we establish the asymptotic convergence of the conditional density. The almost sure convergence is given in the following theorem.

Theorem 4.7. Suppose that (H1)-(H3), (H5)(i-iii) and (H6)(i-iii) are satisfied. If $\lim _{n \rightarrow \infty} n h_{n}^{2}=0$, then

$$
\begin{aligned}
\limsup _{n \rightarrow \infty}\left[\frac{n F\left(h_{n}\right)}{\ln \ln n}\right]^{1 / 2}\left[\widehat{f}_{n}^{[x, l]}(y)-f^{[x]}(y)\right] & \\
=\frac{\left[2 M_{2} \varpi_{[1-2 l]} f^{[x]}(y) \int_{\mathbb{R}} H^{\prime 2}(t) d t\right]^{1 / 2}}{\beta_{[1-l]} M_{1}} & \text { a.s. }
\end{aligned}
$$

The quadratic mean convergence of the conditional density is given in the next theorem.

TheOrem 4.8. Suppose that (H1)-(H3), (H5)(i-iii) and (H6)(i-iii) are satisfied. If there is a constant $c>0$ such that $n F\left(h_{n}\right) h_{n}^{2} \rightarrow c$ as $n \rightarrow \infty$, then

$$
\begin{aligned}
\lim _{n \rightarrow \infty} n F\left(h_{n}\right) E\left[\widehat{f}_{n}^{[x, l]}(y)-f^{[x]}(y)\right]^{2} \\
=\frac{\beta_{[1-2 l]}}{\beta_{[1-l]}^{2}} \frac{M_{2}}{M_{1}^{2}} f^{[x]}(y) \int_{\mathbb{R}} H^{\prime 2}(t) d t+c\left[\phi^{\prime}(0)\right]^{2} \frac{\alpha_{[l]}^{2}}{\beta_{[1-l]}^{2}} \frac{M_{0}^{2}}{M_{1}^{2}} .
\end{aligned}
$$

The proofs of the theorem depend on the following lemmas.

Lemma 4.9. Suppose that (H1)-(H3), (H5)(i-iii) and (H6)(i-iii) are satisfied. If there is a constant $c>0$ such that $n F\left(h_{n}\right) h_{n}^{2} \rightarrow c$ as $n \rightarrow \infty$, then

$$
E\left[\widehat{f}_{n}^{[x, l]}(y)-f^{[x]}(y)\right]=h_{n} \phi^{\prime}(0) \frac{\alpha_{[l]}}{\beta_{[1-l]}} \frac{M_{0}}{M_{1}}[1+o(1)]+O\left[\frac{1}{n F\left(h_{n}\right)}\right] .
$$

The asymptotic bias of the conditional density estimate is equivalent to that of the nonrecursive estimate for dependent data [D08], and is different from the case where the data are independent [BRS15], given as $O\left(\frac{1}{n h_{H} F\left(h_{K}\right)}\right)$. 
Lemma 4.10. Suppose that (H1)-(H3), (H5)(i-iii) and (H6)(i-iii) are satisfied. If there is a constant $c>0$ such that $n F\left(h_{n}\right) h_{n}^{2} \rightarrow c$ as $n \rightarrow \infty$, then

$$
\lim _{n \rightarrow \infty} n F\left(h_{n}\right) \operatorname{Var}\left[\widehat{f}_{n}^{[x, l]}(y)\right]=\frac{\beta_{[1-2 l]}}{\beta_{[1-l]}^{2}} \frac{M_{2}}{M_{1}^{2}} \theta_{\varepsilon}^{2}(X) .
$$

In practice, when we put $c=0$ in Theorem 4.8 the following result holds.

Corollary 4.11. Suppose that (H1)-(H3), (H5)(i-iii) and (H6)(i-iii) are satisfied. If $n F\left(h_{n}\right) h_{n}^{2} \rightarrow 0$ as $n \rightarrow \infty$, then

$$
\lim _{n \rightarrow \infty} n F\left(h_{n}\right) E\left[\widehat{f}_{n}^{[x, l]}(y)-f^{[x]}(y)\right]^{2}=\frac{\beta_{[1-2 l]}}{\beta_{[1-l]}^{2}} \frac{M_{2}}{M_{1}^{2}} f^{[x]}(y) \int_{\mathbb{R}} H^{\prime 2}(t) d t .
$$

Here, the asymptotic variance of our conditional density estimator is different from the nonrecursive one, $\left(O\left(\frac{1}{n h_{H} F\left(h_{K}\right)}\right)\right)$, for both independent [BRS15] and dependent D08] cases.

We can now present the asymptotic properties of our conditional hazard function. Let us begin with the almost sure convergence given in the following theorem.

TheOREM 4.12. Suppose that hypotheses $(\mathrm{H} 1)-(\mathrm{H} 3)$ and $(\mathrm{H} 5)-(\mathrm{H} 8)$ are satisfied. If $\lim _{n \rightarrow \infty} n h_{n}^{2}=0$, then

$$
\limsup _{n \rightarrow \infty}\left[\frac{n F\left(h_{n}\right)}{\ln \ln n}\right]^{1 / 2}\left[\widehat{h}_{n}^{[x, l]}(y)-h^{[x]}(y)\right]=C(x, y) \frac{\sqrt{2 M_{2}}}{M_{1} \beta_{[1-l]}} \quad \text { a.s. }
$$

where

$$
C(x, y)=\frac{\sqrt{\varpi_{[1-2 l]} \theta_{\varepsilon}^{2}(X)}+h^{[x]}(y) \sqrt{\beta_{[1-2 l]} \sigma_{\varepsilon}^{2}(X)}}{1-F^{[x]}(y)} .
$$

Next, the quadratic mean convergence is stated in the following theorem.

THEOREM 4.13. Suppose that (H1)-(H3) and (H5)-(H8) are satisfied. If there exists $c>0$ such that $n F\left(h_{n}\right) h_{n}^{2} \rightarrow c$ as $n \rightarrow \infty$, then

$$
\begin{array}{rl}
\lim _{n \rightarrow \infty} n & F\left(h_{n}\right) E\left[\widehat{h}_{n}^{[x, l]}(y)-h^{[x]}(y)\right]^{2} \\
= & \frac{h^{[x]}(y)^{2}}{\left[1-F^{[x]}(y)\right]^{2}}\left\{M_{2} \beta_{[1-2 l]} \frac{\beta_{[1-2 l]}}{\beta_{[1-l]}^{2}} \frac{M_{2}}{M_{1}^{2}} \sigma_{\varepsilon}^{2}(X)+c\left[\varphi^{\prime}(0)\right]^{2} \frac{\alpha_{[l]}^{2}}{\beta_{[1-l]}^{2}} \frac{M_{0}^{2}}{M_{1}^{2}}\right\} \\
& +\frac{2 h^{[x]}(y)}{\left[1-F^{[x]}(y)\right]^{2}}\left[c \phi^{\prime}(0) \varphi^{\prime}(0) \frac{\alpha_{[l]}^{2}}{\beta_{[1-l]}^{2}} \frac{M_{0}^{2}}{M_{1}^{2}}+\frac{\beta_{[1-2 l]}}{\beta_{[1-l]}^{2}} \frac{M_{2}}{M_{1}^{2}} \sqrt{\theta_{\varepsilon}^{2}(X) \sigma_{\varepsilon}^{2}(X)}\right] \\
& +\frac{1}{\left[1-F^{[x]}(y)\right]^{2}}\left[\frac{\beta_{[1-2 l]}}{\beta_{[1-l]}^{2}} \frac{M_{2}}{M_{1}^{2}} \theta_{\varepsilon}^{2}(X)+c\left[\phi^{\prime}(0)\right]^{2} \frac{\alpha_{[l]}^{2}}{\beta_{[1-l]}^{2}} \frac{M_{0}^{2}}{M_{1}^{2}}\right] .
\end{array}
$$

In practice, by taking $c=0$ in Theorem 4.13 we have the following corollary. 
COROLlary 4.14. Suppose that (H1)-(H3) and (H5)-(H8) are satisfied. If $n F\left(h_{n}\right) h_{n}^{2} \rightarrow 0$ as $n \rightarrow \infty$, then

$$
\begin{aligned}
\lim _{n \rightarrow \infty} n F\left(h_{n}\right) E\left[\widehat{h}_{n}^{[x, l]}(y)-h^{[x]}(y)\right]^{2} & \\
& =\frac{1}{\left[1-F^{[x]}(y)\right]^{2}} \frac{\beta_{[1-2 l]}}{\beta_{[1-l]}^{2}} \frac{M_{2}}{M_{1}^{2}}\left[\theta_{\varepsilon}(X)+h^{[x]}(y) \sigma_{\varepsilon}(X)\right]^{2} .
\end{aligned}
$$

REMARK 4.15. As noticed in ACT14, the choices of bandwidths and small ball probabilities involve that $\beta_{[1-2 l]} / \beta_{[1-l]}^{2}<1$. Consequently, the recursive estimators are more efficient than classical estimators in this case in the sense that their asymptotic variance is small for given $M_{1}$ and $M_{2}$. The bias and variance of our conditional hazard function estimator are both equivalent to $O\left(\frac{1}{n F\left(h_{n}\right)}\right)$ and different from that of the nonrecursive estimator whose bias and variance are both equivalent to $O\left(\frac{1}{n h_{H} F\left(h_{K}\right)}\right)$ for independent [BRS15] and dependent D08 data.

5. Simulation study and a real data application. The performance of our estimator is now checked using a simulation study. Further, in order to show the efficiency of our approach in concrete cases, we present a practical use of our model to study a time series.

5.1. Estimating some parameters in practice. In practice, when we want to build the confidence interval or compute the mean square error of the conditional hazard function, we need to estimate the constants on which the mean square error (see Corollary 4.14) of our estimator depends: $\beta_{[1-2 l]}$, $\beta_{[1-l]}^{2}, M_{1}$ and $M_{2}$.

In Corollary 4.14. $F^{[x]}(y), f^{[x]}(y), \beta_{[1-2 l]}$ and $\beta_{[1-l]}^{2}$ must be replaced by their respective estimators, and $K$ and $H$ must be chosen.

The constants $M_{1}$ and $M_{2}$ are estimated [ACT14 respectively by

$$
\widehat{M}_{1}=\frac{1}{n} \sum_{i=1}^{n} \frac{1}{F\left(h_{i}\right)} K\left(\frac{\left\|x-X_{i}\right\|}{h_{i}}\right), \quad \widehat{M}_{2}=\frac{1}{n} \sum_{i=1}^{n} \frac{1}{F\left(h_{i}\right)} K^{2}\left(\frac{\left\|x-X_{i}\right\|}{h_{i}}\right) .
$$

However, the distribution function $F$ must be chosen.

5.2. As simulation study. To observe the behavior of our recursive estimator in practice, this section gives a simulation study. We compare our recursive estimator and the initial one from [BRS15] defined as

$$
\hat{h}^{[x]}(y)=\frac{\hat{f}^{[x]}(y)}{1-\hat{F}^{[x]}(y)},
$$


where

$$
\begin{aligned}
\widehat{F}^{[x]}(y) & =\frac{\sum_{i=1}^{n} K\left(h_{K}^{-1} d\left(x, X_{i}\right)\right) H\left(h_{H}^{-1}\left(y-Y_{i}\right)\right)}{\sum_{i=1}^{n} K\left(h_{K}^{-1} d\left(x, X_{i}\right)\right)}, \\
\widehat{f}^{[x]}(y) & =\frac{\sum_{i=1}^{n} K\left(h_{K}^{-1} d\left(x, X_{i}\right)\right) H^{\prime}\left(h_{H}^{-1}\left(y-Y_{i}\right)\right)}{h_{H} \sum_{i=1}^{n} K\left(h_{K}^{-1} d\left(x, X_{i}\right)\right)} .
\end{aligned}
$$

We simulate our data in the following way: The functional variable $X$ is supposed to be generated by the equation

$$
X(t)=A(2-\cos (\pi t W))+(1-A) \cos (\pi t W), \quad t \in[0,1],
$$

where $W \rightsquigarrow \mathcal{N}(0,1)$ and $A$ is a random variable Bernoulli distributed with parameter $p=1 / 2$. The curves $X_{1}, \ldots, X_{n}$ for $n=122$ are plotted in Figure 1 .

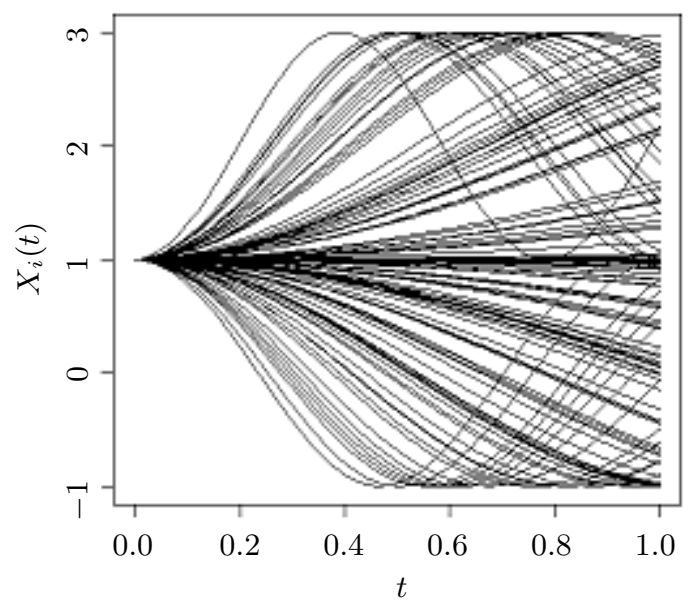

Fig. 1. The curves $\left\{X_{i}(t)\right\}_{i=1}^{122}, t \in[0,1]$

As the curves are smooth, the $L_{2}$ distance between the second derivatives of the curves can be utilized as a semi-metric. We simulate the values of $Y$ by the relation $Y=R(x)+\epsilon=\int_{0}^{1} x^{\prime}(s)^{2} d s+\epsilon$. The error $\epsilon$ is simulated as a Gaussian random variable with mean 0 and standard deviation 0.1. Note that with this model definition, the conditional distribution of $Y_{i}$ given $X_{i}=x$ is the Gaussian distribution $\mathcal{N}(R(x), 0.01)$, thus we may explicitly get the conditional hazard function $h^{[X]}(\cdot)$.

In this investigation, the proposed estimator depends on the choice of various parameters, namely, the semi-norm $\|\cdot\|$ of the function space $\mathcal{F}$, the sequence $\left(h_{n}\right)$ of bandwidths, the kernel $K$, the parameter $l$ and the distribution function $F$ when $l \neq 0$. And as the choice of $K$ is not important, we employed the quadratic kernel defined by $K(u)=\left(1-u^{2}\right) \mathbb{I}_{[0,1]}(u)$ for 
all $u \in \mathbb{R}$, which behaves properly in practice and is easy to implement, and we put $H^{\prime}=K$. We estimated the distribution function $F$ by using the empirical distribution function, which is uniformly convergent. For the choice of the parameter $l$, we computed the mean square errors of our recursive estimators for some values $l$ in $\{0,1 / 2,1\}$. The parameter $l$ has shown a negligible influence on the mean square error (MSE) of our estimator, for 500 simulations we obtained approximately the same value $(\mathrm{MSE}=0.0050)$. Thus, we take $l=0$.

- Choice of the bandwidth. In this simulation, the semi-metric (seminorm) was based on the second derivatives of the curves; the shape of the curves (Figure 1) permits us to utilize

$$
\left\|x-x^{\prime}\right\|=\left(\int_{0}^{1}\left(x^{(i)}(t)-x^{(i)}(t)\right)^{2} d t\right)^{1 / 2},
$$

where $x^{(i)}$ is the $i$ th derivative of the curve $x(\cdot)$; here we take $i=2$. Moreover, as indicated in ACT14, the choice of the semi-norm depends on the treated data. Then, as pointed out in [LM14, there is no automatic data-driven method for selecting bandwidths when estimating a conditional hazard function in the functional case. Consequently, for our investigation we consider the bandwidth selector associated to the conditional density estimation presented as

$$
\begin{aligned}
\mathrm{CV} f_{Y / X}(h)= & \frac{1}{n} \sum_{i=1}^{n} W_{1}\left(X_{i}\right) \int \widehat{f}_{n}^{\left[X_{i}, l\right]^{2}}(y) W_{2}(y) d y \\
& \left.-\frac{2}{n} \sum_{i=1}^{n} \widehat{f}_{n}^{\left[X_{i}, l\right]}\right]^{-i}\left(Y_{i}\right) W_{1}\left(X_{i}\right) W_{2}\left(Y_{i}\right),
\end{aligned}
$$

where $W_{1}$ and $W_{2}$ are some suitable trimming functions and

$$
\widehat{f}_{n}^{\left[X_{i}, l\right]^{-i}}(y)=\frac{\sum_{j \neq i} \frac{1}{h_{j}\left[F\left(h_{j}\right)\right]^{l}} K\left(\frac{\left\|x-X_{j}\right\|}{h_{j}}\right) H^{\prime}\left(\frac{y-Y_{j}}{h_{j}}\right)}{\sum_{j \neq i} \frac{1}{\left[F\left(h_{j}\right)\right]^{l}} K\left(\frac{\left\|x-X_{j}\right\|}{h_{j}}\right)},
$$

and we use it for the conditional hazard function. As mentioned in [LM14, the optimal bandwidth is locally selected among the $k$ nearest neighbors (for more discussion see [BFRV07]). For the conditional distribution function we can use the following cross-validation rule:

$$
\mathrm{CV} F_{Y / X}(h)=\frac{1}{n} \sum_{i=1}^{n} \int\left[\mathbb{I}_{Y_{i} \leq y}-\widehat{F}_{n}^{\left[X_{i}, l\right]^{-i}}(y)\right]^{2} W(y) d y,
$$

where $W$ is a suitable trimming function and 


$$
\widehat{F}_{n}^{\left[X_{i}, l\right]^{-i}}(y)=\frac{\sum_{j \neq i} \frac{1}{\left[F\left(h_{j}\right)\right]^{l}} K\left(\frac{\left\|x-X_{j}\right\|}{h_{j}}\right) H\left(\frac{y-Y_{j}}{h_{j}}\right)}{\sum_{j \neq i} \frac{1}{\left[F\left(h_{j}\right)\right]^{l}} K\left(\frac{\left\|x-X_{j}\right\|}{h_{j}}\right)} .
$$

To facilitate calculations, as $\mathbb{E}(Y \mid X=x)=\int y f^{[x]}(y) d y$, and a natural estimator of the regression function is $\int y \widehat{f}_{n}^{[x, l]}(y) d y$, we propose to use the criterion

$$
\mathrm{CV}(h)=\frac{1}{n} \sum_{i=1}^{n}\left(Y_{i}-\int y \widehat{f}_{n}^{\left[X_{i}, l\right]^{-}}{ }^{-}(y) d y\right)^{2} .
$$

For our recursive estimator, we use a sequence of bandwidths $h_{i}=$ $C \max _{i=1, \ldots, n}\left\|X_{i}-x\right\| i^{-v}=h_{i}(C, v)$ with $C \in\{1,1.5,2,4\}$ and $v \in\left\{\frac{1}{10}, \frac{1}{9}, \frac{1}{7}\right.$, $\left.\frac{1}{3}, \frac{1}{2}, 1\right\}$. We choose $C$ and $v$ that minimize $\mathrm{CV}(C, v)$, we found $C=2$ and $v=\frac{1}{10}$. For the nonrecursive method we used a global bandwidth selection $\left(h_{K}=h_{H}=h\right.$ ) with a cross-validation procedure on the $k$-nearest neighbors.

- Comparative tools. To examine the performance of $\widehat{h}_{n}^{[x, l]}(y)$, we randomly split the data into two subsets, a training sample $\left(X_{i}, Y_{i}\right)_{i \in I}(100$ locations) and a test sample $\left(X_{i}, Y_{i}\right)_{i \in J}$ (22 locations), then we utilize an empirical version of the global mean squared error (MSE) and mean absolute error (MAE),

$$
\begin{aligned}
\text { MSE } & =\frac{1}{22} \sum_{i \in J}\left(h^{\left[X_{i}\right]}\left(Y_{i}\right)-\widehat{h}_{n}^{\left[X_{i}, l\right]}\left(Y_{i}\right)\right)^{2}, \\
\text { MAE } & =\frac{1}{22} \sum_{i \in J}\left|h^{\left[X_{i}\right]}\left(Y_{i}\right)-\widehat{h}_{n}^{\left[X_{i}, l\right]}\left(Y_{i}\right)\right|,
\end{aligned}
$$

as accuracy measures.

Table 1. MAE and MSE computed via 500 simulations for different values of $n$ with the optimal values of bandwidth provided by $C_{\mathrm{CV}}$ and $v_{\mathrm{CV}}$.

\begin{tabular}{cccc}
\hline & $n=122$ & $n=178$ & $n=478$ \\
\hline$(1)$ & 0.0812 & 0.0625 & 0.0462 \\
& 0.0085 & 0.0043 & 0.0023 \\
\hline$(2)$ & 0.0805 & 0.0623 & 0.0459 \\
& 0.0084 & 0.0042 & 0.0023 \\
\hline
\end{tabular}

As is seen in Table 1 (where (1) represents MAE and MSE of our recursive estimator and (2) represents those of the nonrecursive one) for different values of $n$, the classical estimator is better than the recursive one. Its MSE and MAE are smaller, but there is not a great difference between the behavior of the two methods. In fact, the difference in terms of MSE (as appearing in the table) is very small. Moreover, in order to check the behavior of the prediction errors when the sample size increases, we used $n=122, n=178$ 
and $n=478$. Then, as expected, the errors decreased when the sample size increased.

- Computation times. Here we emphasize a significant advantage of the recursive estimator over the nonrecursive one, when new values of the explanatory variable are sequentially added to the database. As pointed out in ACT14, when a new observation $\left(X_{n+1}, Y_{n+1}\right)$ appears, the computation of the recursive estimator $\widehat{h}_{n}^{[x, l]}(y)$ requires another iteration of the algorithm via its value computed with the sequence $\left(X_{i}, Y_{i}\right)_{i=1}^{n}$, while the initial estimator must be recalculated via the complete sample $\left(X_{i}, Y_{i}\right)_{i=1}^{n+1}$.

Next, we illustrate the computation time difference between the two estimators in such situations. From an initial sample $\left(X_{i}, Y_{i}\right)_{i=1}^{n}$ of size $n=122$, we consider $N$ additional observations for different values of $N$. We compare the cumulative computation times to get the recursive and the classical estimators when these new observations are added. The characteristics of the computer we used to perform these computations are CPU: Intel Celeron N2830 $2.60 \mathrm{GHz}, \mathrm{HD}: 250 \mathrm{G}$, Memory: $3.23 \mathrm{G}$. The curves $X_{1}, \ldots, X_{n}$ as well as the new observations $X_{n+1}, \ldots, X_{n+N}$ are computed via (5.2) with $n=122$ and $N \in\{1,28,78,178,478\}$. The semi-norm, the bandwidth sequence and the parameter $l$ are selected as previously. The computation times are listed in Table 2. Here, our estimator shows a significant preference as regards computation time compared to the nonrecursive estimator.

Table 2. Cumulative computation times in seconds for the recursive and nonrecursive estimators when adding $N$ new observations for different values of $N$.

\begin{tabular}{cccccc}
\hline$N$ & 1 & 28 & 78 & 178 & 478 \\
\hline Comp. time for $\widehat{h}_{n+1}^{[x, l]}$ & 0.840 & 1.050 & 1.260 & 1.260 & 2.490 \\
\hline Comp. time for $\widehat{h}_{n+1}^{[x]}$ & 1.640 & 3.680 & 18.150 & 75.600 & 375.520 \\
\hline
\end{tabular}

5.3. Real data application. In this subection we examine the efficiency of our procedure in a real dataset study. The functional data are particulary suitable for analyzing time series. We illustrate this via the El Niño time series $\left({ }^{1}\right)$ that furnishes the monthly sea surface temperature from January 1982 to December 2016 (420 months). From this time series, we extracted 34 annual curves $X_{1}, \ldots, X_{34}$ from 1982 to 2016 , discretized into $p=12$ points. These curves are presented in Figure 2.

The variable of interest at month $j$ of year $i$ is the sea temperature $X_{i+1}$ for month $j$. Namely, for $j=1, \ldots, 12$ and $i=1, \ldots, 34$, we have $Y_{i}^{[j]}=$ $X_{i+1}(j)$. We predicted the values of $Y_{34}^{[1]}, \ldots, Y_{34}^{[12]}$ (that is, the values of the

$\left({ }^{1}\right)$ Available at http://www.cpc.ncep.noaa.gov/data/indices/ 
curve $\left.X_{35}\right)$ via conditional mode, using the routine npfda $\left({ }^{2}\right)$. The estimators (recursive and nonrecursive) were computed by selecting the semi-norm, the bandwidth sequence and the parameter $l$ as previously.

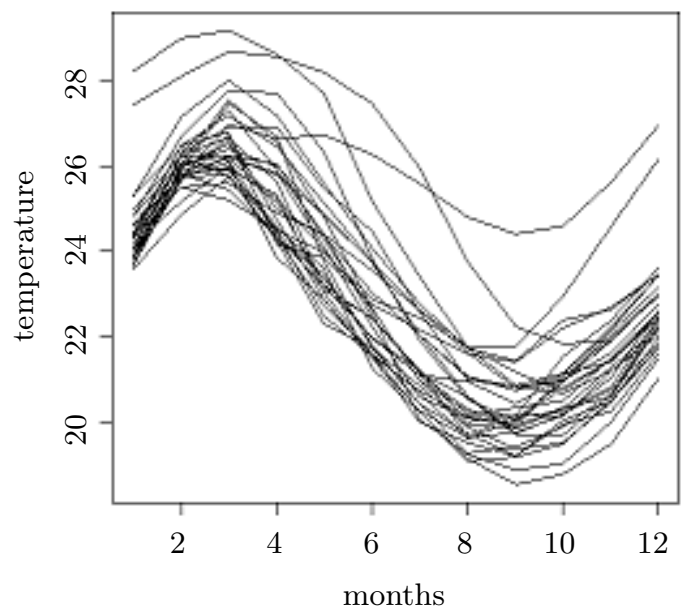

Fig. 2. El Niño annual temperature curves from 1982 to 2016

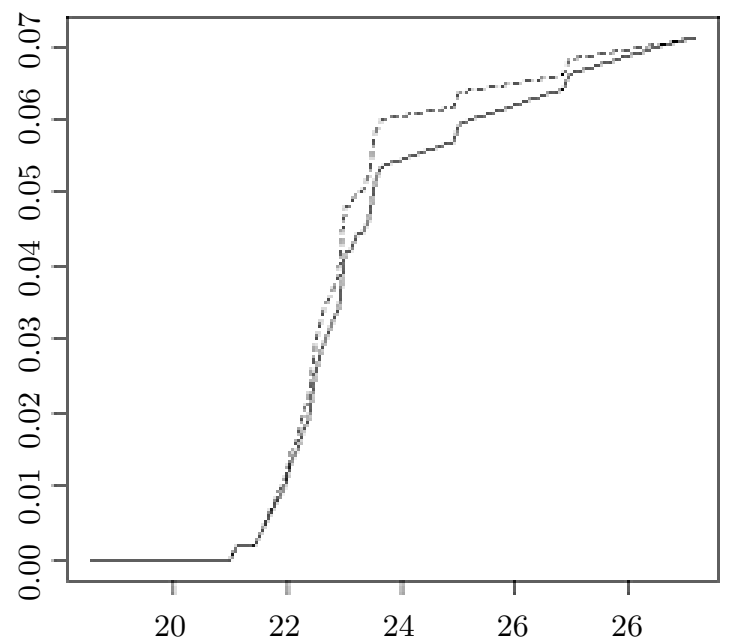

Fig. 3. Curves of the two conditional hazard functions. The solid line represents our recursive conditional hazard function, the dashed line denotes the nonrecursive one.

The probabilities that the time series take some predicted values $\widehat{Y}_{34}^{[1]}, \ldots$ $\ldots, \widehat{Y}_{34}^{[12]}$ are given by the conditional densities $\widehat{f}_{n}^{\left[X_{34}\right]}\left(\widehat{Y}_{34}^{[1]}\right), \ldots, \widehat{f}_{n}^{\left[X_{34}\right]}\left(\widehat{Y}_{34}^{[12]}\right)$. The instantaneous risks are $\widehat{h}_{n}^{\left[X_{34}\right]}\left(\widehat{Y}_{34}^{[1]}\right), \ldots, \widehat{h}_{n}^{\left[X_{3}\right]}\left(\widehat{Y}_{34}^{[12]}\right)$. We analyzed the

$\left({ }^{2}\right)$ Available at http://www.math.univ-toulouse.fr/staph/npfda/. 
mean square prediction error through 2016 and obtained MSPE $=0.00025$ for the recursive estimator, and MSPE $=0.00011$ for the nonrecursive one. The classical nonparametric (nonrecursive) estimator once more showed its advantage as regards prediction, while our estimator behaved well and had an advantage regarding computation time. The computation time (in seconds) required for our estimator to predict twelve values (the last year) was $0.020 \mathrm{~s}$, while for the nonrecursive estimator the time was $0.250 \mathrm{~s}$. We plot the two hazard functions in Figure 3 .

It is difficult to decide which curve is smoother, even if the nonrecursive estimator (dashed curve) seems to be slightly smoother compared to the recursive one. We conclude that even if the nonrecursive estimator showed a small advantage over our estimator with regard to the mean prediction error, there is not a big difference between their behavior, and our recursive estimator has an advantage as regards computation time.

\section{Proofs}

Proof of Theorem 4.1. Note that $\widehat{F}_{n}^{[x, l]}(y)$ can be written as

$$
\widehat{F}_{n}^{[x, l]}(y)=\frac{\varphi_{n}^{[x, l]}(y)}{f_{n}^{[l]}(x)},
$$

where $\varphi_{n}^{[x, l]}(y)$ and $f_{n}^{[l]}(x)$ are defined above. Let

$$
\widehat{F}_{n}^{[x, l]}(y)-F^{[x]}(y)=\frac{\varphi_{n}^{[x, l]}(y)-F^{[x]}(y) f_{n}^{[l]}(x)}{f_{n}^{[l]}(x)} .
$$

The main idea is to show that $f_{n}^{[l]}(x)$ converges almost surely to $f^{[l]}(x)$ and that $\varphi_{n}^{[x, l]}(y)-F^{[x]}(y) f_{n}^{(l)}(x)$ converges almost surely to 0 .

1. Firstly, we prove that $\varphi_{n}^{[x, l]}(y)-F^{[x]}(y) f_{n}^{(l)}(x)$ converges almost surely to 0 .

We can write

$$
\begin{aligned}
\varphi_{n}^{[x, l]}(y)-F^{[x]}(y) f_{n}^{[l]}(x) & \\
= & \left\{\varphi_{n}^{[x, l]}(y)-F^{[x]}(y) f_{n}^{[l]}(y)-E\left[\varphi_{n}^{[x, l]}(y)-F^{[x]}(y) f_{n}^{[l]}(x)\right]\right\} \\
& +\left\{E\left[\varphi_{n}^{[x, l]}(y)-F^{[x]}(y) f_{n}^{[l]}(x)\right]\right\} \\
= & I_{1}+I_{2} .
\end{aligned}
$$

Studying $I_{1}$. Let

$$
\begin{aligned}
W_{i} & =\frac{1}{\left[F\left(h_{i}\right)\right]^{l}} K\left(\frac{\left\|x-X_{i}\right\|}{h_{i}}\right)\left[H\left(\frac{y-Y_{i}}{h_{i}}\right)-F^{[x]}(y)\right], \\
Z_{i} & =W_{i}-E\left(W_{i}\right), \quad S_{n}=\sum_{i=1}^{n} Z_{i} .
\end{aligned}
$$


Note that

$$
I_{1}=\frac{S_{n}}{\sum_{i=1}^{n}\left[F\left(h_{i}\right)\right]^{1-l}}
$$

Putting $V_{n}=\sum_{i=1}^{n} E Z_{i}^{2}$, we get

$$
\begin{aligned}
V_{n}= & \sum_{i=1}^{n} \operatorname{Var}\left(W_{i}\right) \\
= & \sum_{i=1}^{n}\left[F\left(h_{i}\right)\right]^{-2 l}\left\{E\left(K^{2}\left(\frac{\left\|x-X_{i}\right\|}{h_{i}}\right)\left[H\left(\frac{y-Y_{i}}{h_{i}}\right)-F^{[x]}(y)\right]^{2}\right)\right\} \\
& -\sum_{i=1}^{n}\left[F\left(h_{i}\right)\right]^{-2 l} E^{2}\left(K\left(\frac{\left\|x-X_{i}\right\|}{h_{i}}\right)\left[H\left(\frac{y-Y_{i}}{h_{i}}\right)-F^{[x]}(y)\right]\right) \\
= & A_{1}-A_{2} .
\end{aligned}
$$

We have

$$
A_{1}=\sum_{i=1}^{n}\left[F\left(h_{i}\right)\right]^{-2 l} E\left\{K^{2}\left(\frac{\left\|x-X_{i}\right\|}{h_{i}}\right) E\left(\left[H\left(\frac{y-Y_{i}}{h_{i}}\right)-F^{[x]}(y)\right]^{2} \mid X_{i}\right)\right\} .
$$

Now

$$
\begin{gathered}
E\left(\left[H\left(\frac{y-Y_{i}}{h_{i}}\right)-F^{[x]}(y)\right]^{2} \mid X_{i}\right) \\
\quad=\operatorname{Var}\left(\left[H\left(\frac{y-Y_{i}}{h_{i}}\right)\right] \mid X_{i}\right)+E^{2}\left(\left[H\left(\frac{y-Y_{i}}{h_{i}}\right)-F^{[x]}(y)\right] \mid X_{i}\right) \\
=\sigma_{\varepsilon i}^{2}(X)+E^{2}\left(\left[H\left(\frac{y-Y_{i}}{h_{i}}\right)-F^{[x]}(y)\right] \mid X_{i}\right) .
\end{gathered}
$$

Since

$$
\begin{aligned}
E\left(\left[H\left(\frac{y-Y_{i}}{h_{i}}\right)-F^{[x]}(y)\right] \mid X_{i}\right) & =\int_{\mathbb{R}} H^{\prime}(t)\left[F^{\left[X_{i}\right]}\left(y-h_{i} t\right)-F^{\left[X_{i}\right]}(y)\right] d t \\
& +\int_{\mathbb{R}} H^{\prime}(t)\left[F^{\left[X_{i}\right]}(y)-F^{[x]}(y)\right] d t \\
& =o\left(h_{i}^{b_{2}-1}\right)+\left[F^{\left[X_{i}\right]}(y)-F^{[x]}(y)\right] \quad(\mathrm{H} 5)(\mathrm{i}-\mathrm{ii}),
\end{aligned}
$$

we get

$E\left(\left[H\left(\frac{y-Y_{i}}{h_{i}}\right)-F^{[x]}(y)\right]^{2} \mid X_{i}\right)=o\left(h_{i}^{b_{2}-1}\right)+\sigma_{\varepsilon i}^{2}(X)+\left[F^{\left[X_{i}\right]}(y)-F^{[x]}(y)\right]^{2}$.

Consequently, 


$$
\begin{aligned}
& E\left(K^{2}\left(\frac{\left\|x-X_{i}\right\|}{h_{i}}\right)[H(\right.\left.\left.\left.\frac{y-Y_{i}}{h_{i}}\right)-F^{[x]}(y)\right]^{2}\right) \\
&=\left\{o\left(h_{i}^{b_{2}-1}\right)+\sigma_{\varepsilon i}^{2}(X)\right\} E\left[K^{2}\left(\frac{\left\|x-X_{i}\right\|}{h_{i}}\right)\right] \\
&+E\left[\left\{F^{\left[X_{i}\right]}(y)-F^{[x]}(y)\right\}^{2} K^{2}\left(\frac{\left\|x-X_{i}\right\|}{h_{i}}\right)\right] .
\end{aligned}
$$

Let

$$
E\left[K^{2}\left(\frac{\left\|x-X_{i}\right\|}{h_{i}}\right)\right]=F\left(h_{i}\right)\left[K^{2}(1)-\int_{0}^{1}\left(K^{2}(s)\right)^{\prime} \tau_{h_{i}}(s) d s\right]
$$

Next, by using (H3)(ii), (H6)(i), 6.1) and Toeplitz' Lemma we find that

$$
\begin{aligned}
\frac{\sum_{i=1}^{n}\left[F\left(h_{i}\right)\right]^{-2 l}\left\{o\left(h_{i}^{b_{2}-1}\right)+\sigma_{\varepsilon i}^{2}(X)\right\} E\left[K^{2}\left(\frac{\left\|x-X_{i}\right\|}{h_{i}}\right)\right]}{n\left[F\left(h_{n}\right)\right]^{1-2 l}} & \stackrel{n \rightarrow \infty}{\longrightarrow} \beta_{[1-2 l]} F^{[x]}(y)\left(1-F^{[x]}(y)\right) M_{2} .
\end{aligned}
$$

Using (H5)(ii) we get

$$
\begin{aligned}
0 & \leq E\left[\left\{F^{\left[X_{i}\right]}(y)-F^{[x]}(y)\right\}^{2} K^{2}\left(\frac{\left\|x-X_{i}\right\|}{h_{i}}\right)\right] \\
& \leq E\left[\left\|x-X_{i}\right\|^{2 b_{1}} K^{2}\left(\frac{\left\|x-X_{i}\right\|}{h_{i}}\right)\right] .
\end{aligned}
$$

But

$$
\begin{gathered}
E\left[\left\|x-X_{i}\right\|^{2 b_{1}} K^{2}\left(\frac{\left\|x-X_{i}\right\|}{h_{i}}\right)\right] \\
\leq E\left[\sup _{X_{i} \in B\left(x, h_{i}\right)}\left\|x-X_{i}\right\|^{2 b_{1}} K^{2}\left(\frac{\left\|x-X_{i}\right\|}{h_{i}}\right)\right] \\
\leq h_{i}^{2 b_{1}} E\left[K^{2}\left(\frac{\left\|x-X_{i}\right\|}{h_{i}}\right)\right],
\end{gathered}
$$

where $B\left(x, h_{i}\right)=\left\{x^{\prime} \in \mathcal{F} \mid\left\|x-x^{\prime}\right\| \leq h_{i}\right\}$. Then, via 6.1),

$$
0 \leq \frac{\sum_{i=1}^{n}\left[F\left(h_{i}\right)\right]^{-2 l} h_{i}^{2 b_{1}} E\left[K^{2}\left(\frac{\left\|x-X_{i}\right\|}{h_{i}}\right)\right]}{n\left[F\left(h_{n}\right)\right]^{1-2 l}} \stackrel{n \rightarrow \infty}{\longrightarrow} 0 .
$$

Now, using 6.2 and 6.3), we obtain

$$
\frac{A_{1}}{n\left[F\left(h_{n}\right)\right]^{1-2 l}} \stackrel{n \rightarrow \infty}{\longrightarrow} \beta_{[1-2 l]} F^{[x]}(y)\left(1-F^{[x]}(y)\right) M_{2} .
$$


Similarly to (6.3), when (H6)(i) is satisfied we get

$$
\frac{A_{2}}{n\left[F\left(h_{n}\right)\right]^{1-2 l}} \underset{n \rightarrow \infty}{\longrightarrow} 0 \text {. }
$$

Therefore, $V_{n} \sim n\left[F\left(h_{n}\right)\right]^{1-2 l} \beta_{[1-2 l]} F^{[x]}(y)\left(1-F^{[x]}(y)\right) M_{2}$ as $n \rightarrow \infty$. By assuming that $n\left[F\left(h_{n}\right)\right] \rightarrow \infty$ we obtain also $\ln F\left(h_{n}\right) / \ln n \rightarrow 0$. By (H4), we deduce that

$$
\lim _{n \rightarrow \infty} \frac{n F\left(h_{n}\right)(\ln n)^{-2 / \mu}}{\ln \left[n\left(F\left(h_{n}\right)\right)^{1-2 l}\right]\left\{\ln \ln \left[n\left(F\left(h_{n}\right)\right)^{1-2 l}\right]\right\}^{2(\alpha+1)}}=\infty .
$$

Now, let $b_{n}=(\delta \ln n)^{1 / \mu}$ with $\delta>0$. There exists $n_{0} \geq 1$ such that for all $i \geq n_{0}$

$$
\begin{aligned}
& \frac{i F\left(h_{i}\right)(\ln i)^{-2 / \mu}}{\ln \left[i\left(F\left(h_{i}\right)\right)^{1-2 l}\right]\left\{\ln \ln \left[i\left(F\left(h_{i}\right)\right)\right]\right\}^{2(\alpha+1)}} \\
& \quad>\frac{2\|K\|_{\infty}^{2} \max \left\{\left|F^{x}(y)\right|^{2},(\delta \ln i)^{2 / \mu}\right\}}{\left[F\left(h_{i}\right)\right]^{2 l}} \geq Z_{i}^{2},
\end{aligned}
$$

as the event

$$
\left\{Z_{i}^{2}>\frac{i\left[F\left(h_{i}\right)\right]^{1-2 l}}{\ln \left[i\left(F\left(h_{i}\right)\right)^{1-2 l}\right]\left\{\ln \ln \left[i\left(F\left(h_{i}\right)\right)\right]\right\}^{2(\alpha+1)}}\right\}
$$

is impossible for $i \geq n_{0}$.

From $V_{n} \sim n\left[F\left(h_{n}\right)\right]^{1-2 l} \beta_{[1-2 l]} F^{[x]}(y)\left(1-F^{[x]}(y)\right) M_{2}$, we deduce that

$$
\sum_{i=1}^{n} \frac{\left(\ln \ln V_{i}\right)^{\alpha}}{V_{i}} E\left(Z_{i}^{2} \mathbf{1}_{\left\{\frac{V_{i}}{\ln \left[V_{i}\left\{\ln \ln \left[V_{i}\right]\right\}^{2(\alpha+1)}\right.}\right\}}\right)<\infty .
$$

Let $S$ be a random function defined on $[0, \infty[$, where

$$
S(t)=S_{n} \quad \text { for } t \in\left[V_{n}, V_{n+1}[.\right.
$$

By the law of the iterated logarithm, there exists a Brownian motion $\xi$ such that

$$
\left|\frac{S(t)-\xi(t)}{(2 t \ln \ln t)^{1 / 2}}\right|=o\left[(\ln \ln t)^{-\alpha / 2}\right], \quad \forall t \in\left[V_{n}, V_{n+1}[.\right.
$$

But since Brownian motion satisfies the law of the iterated logarithm, we have

$$
\varlimsup_{t \rightarrow \infty} \frac{S(t)}{(2 t \ln \ln t)^{1 / 2}}=\varlimsup_{t \rightarrow \infty}\left[\frac{S(t)-\xi(t)}{(2 t \ln \ln t)^{1 / 2}}+\frac{\xi(t)}{(2 t \ln \ln t)^{1 / 2}}\right]=1 \quad \text { a.s. }
$$

We have $S_{n} /\left(2 V_{n} \ln \ln V_{n}\right)^{1 / 2} \rightarrow 1$ a.s. By using the fact that $S_{n}=$ $I_{1} \sum_{i=1}^{n}\left[F\left(h_{i}\right)\right]^{1-l}$ and $V_{n+1} / V_{n} \rightarrow 1$, we obtain

$$
\varlimsup_{n \rightarrow \infty} \frac{\sum_{i=1}^{n}\left[F\left(h_{i}\right)\right]^{1-l} I_{1}}{\left(2 V_{n} \ln \ln V_{n}\right)^{1 / 2}} \frac{n\left(F\left(h_{n}\right)\right)^{1-2 l}\left\{\ln \ln \left[n\left(F\left(h_{n}\right)\right)^{1-2 l}\right]\right\}^{1 / 2}}{n\left(F\left(h_{n}\right)\right)^{1-2 l}\left\{\ln \ln \left[n\left(F\left(h_{n}\right)\right)^{1-2 l}\right]\right\}^{1 / 2}}=1 \quad \text { a.s. }
$$


But $\sum_{i=1}^{n}\left[F\left(h_{i}\right)\right]^{1-l}=B_{n,(1-l)} n\left[F\left(h_{n}\right)\right]^{1-l}$. We have

$$
\frac{\left\{\ln \ln \left[n\left(F\left(h_{n}\right)\right)^{1-2 l}\right]\right\}^{1 / 2} B_{n,(1-l)}}{\left(2 V_{n} \ln \ln V_{n}\right)^{1 / 2}} \underset{n \rightarrow \infty}{\longrightarrow} \beta_{[1-l]}\left\{2 \beta_{[1-2 l]} F^{[x]}(y)\left(1-F^{[x]}(y)\right) M_{2}\right\}^{1 / 2} .
$$

This yields

$$
\varlimsup_{n \rightarrow \infty}\left\{\frac{n F\left(h_{n}\right)}{\ln \ln \left[n\left(F\left(h_{n}\right)\right)^{1-2 l}\right]}\right\}^{1 / 2} I_{1}=\sigma_{l} \quad \text { a.s. }
$$

where $\sigma_{l}=\left\{2 \beta_{[1-2 l]} F^{[x]}(y)\left(1-F^{[x]}(y)\right) M_{2}\right\}^{1 / 2} / \beta_{[1-l]}$.

As $\ln \ln \left[n\left(F\left(h_{n}\right)\right)^{1-2 l}\right]=(\ln \ln n)[1+o(1)]$, we conclude that

$$
\varlimsup_{n \rightarrow \infty}\left\{\frac{n F\left(h_{n}\right)}{\ln \ln n}\right\}^{1 / 2} I_{1}=\frac{\left\{2 \beta_{[1-2 l]} F^{[x]}(y)\left(1-F^{[x]}(y)\right) M_{2}\right\}^{1 / 2}}{\beta_{[1-l]}} .
$$

Studying $I_{2}$. We have to prove that

$$
\varlimsup_{n \rightarrow \infty}\left\{\frac{n F\left(h_{n}\right)}{\ln \ln n}\right\}^{1 / 2} I_{2}=0 .
$$

We have

$$
\begin{aligned}
I_{2}= & E\left[\varphi_{n}^{[x, l]}(y)-F^{[x]}(y) f_{n}^{[l]}(x)\right] \\
= & \frac{1}{\sum_{i=1}^{n}\left[F\left(h_{i}\right)\right]^{1-l}} \sum_{i=1}^{n} \frac{1}{\left[F\left(h_{i}\right)\right]^{l}} E\left\{\left[H\left(\frac{y-Y_{i}}{h_{i}}\right)-F^{[x]}(y)\right] K\left(\frac{\left\|x-X_{i}\right\|}{h_{i}}\right)\right\} \\
= & \frac{1}{\sum_{i=1}^{n}\left[F\left(h_{i}\right)\right]^{1-l}} \sum_{i=1}^{n} \frac{1}{\left[F\left(h_{i}\right)\right]^{l}} \\
& \times\left\{h_{i} \varphi^{\prime}(0) F\left(h_{i}\right)\left[K(1)-\int_{0}^{1}(s K(s))^{\prime} \tau_{h_{i}}(s) d s\right]+o\left(h_{i}\right)\right\} .
\end{aligned}
$$

The last equality was obtained as follows. Via (H6)(iii), we get evidently

$$
\begin{aligned}
E\left\{\left[H\left(\frac{y-Y_{i}}{h_{i}}\right)-F^{[x]}(y)\right] K\left(\frac{\left\|x-X_{i}\right\|}{h_{i}}\right)\right\} & \\
=E\left[\varphi\left(\left\|x-X_{i}\right\|\right) K\left(\frac{\left\|x-X_{i}\right\|}{h_{i}}\right)\right] & =\int_{0}^{1} \varphi\left(h_{i} t\right) K(t) d P^{\left\|x-x_{i}\right\| / h_{i}}(t) .
\end{aligned}
$$

Using the Taylor expansion for $\varphi$ around 0 , we obtain

$$
\begin{aligned}
E\left\{\left[H\left(\frac{y-Y_{i}}{h_{i}}\right)-F^{[x]}(y)\right]\right. & \left.K\left(\frac{\left\|x-X_{i}\right\|}{h_{i}}\right)\right\} \\
& =h_{i} \varphi^{\prime}(0) \int_{0}^{1} t K(t) d P^{\left\|x-x_{i}\right\| / h_{i}}(t)+o\left[h_{i}\right] .
\end{aligned}
$$


Now, using (H1) and Fubini's Theorem, we get

$$
\int_{0}^{1} t K(t) d P^{\left\|x-x_{i}\right\| / h_{i}}(t)=F\left(h_{i}\right)\left[K(1)-\int_{0}^{1}(s K(s))^{\prime} \tau_{h_{i}}(s) d s\right] .
$$

Then, via hypothesis (H3), as $n \rightarrow \infty$, we have

$$
I_{2} \simeq h_{n} \varphi^{\prime}(0) \frac{\alpha_{[l]}}{\beta_{[1-l]}} M_{0}[1+o(1)] .
$$

Thus

$$
\left\{\frac{n F\left(h_{n}\right)}{\ln \ln n}\right\}^{1 / 2} I_{2}=\left\{\frac{n F\left(h_{n}\right)}{\ln \ln n}\right\}^{1 / 2} h_{n} \varphi^{\prime}(0) \frac{\alpha_{[l]}}{\beta_{[1-l]}} M_{0}[1+o(1)]=o(1)
$$

when $\lim _{n \rightarrow \infty} n h_{n}^{2}=0$. Thus we conclude

$$
\varlimsup_{n \rightarrow \infty}\left\{\frac{n F\left(h_{n}\right)}{\ln \ln n}\right\}^{1 / 2} I_{2}=0
$$

Thus

$$
\begin{aligned}
\left\{\frac{n F\left(h_{n}\right)}{\ln \ln n}\right\}^{1 / 2}\left[\varphi_{n}^{[x, l]}(y)-F^{[x]}(y)\right. & \left.f_{n}^{[l]}(x)\right] \\
& \rightarrow \frac{\left\{2 \beta_{[1-2 l]} F^{[x]}(y)\left(1-F^{[x]}(y)\right) M_{2}\right\}^{1 / 2}}{\beta_{[1-l]}} .
\end{aligned}
$$

2. To finish the proof, we have to show the almost sure convergence of $f_{n}^{[l]}(x)$ towards $f^{[l]}(x)$.

In the same way, putting

$$
\begin{aligned}
W_{i}^{\prime} & =\frac{1}{\left[h_{i} F\left(h_{i}\right)\right]^{l}} K\left(\frac{\left\|x-X_{i}\right\|}{h_{i}}\right)\left[H^{\prime}\left(\frac{y-Y_{i}}{h_{i}}\right)-f^{[x]}(y)\right], \\
Z_{i}^{\prime} & =W_{i}^{\prime}-E\left(W_{i}^{\prime}\right), \quad S_{n}=\sum_{i=1}^{n} Z_{i}^{\prime},
\end{aligned}
$$

we can prove that

$$
f_{n}^{[l]}(x)-E\left[f_{n}^{[l]}(x)\right]=O\left(\sqrt{\frac{\ln \ln n}{n F\left(h_{n}\right)}}\right) \quad \text { a.s. }
$$

As $E\left[f_{n}^{[l]}(x)\right]=M_{1}[1+o(1)]$, it now follows that $f_{n}^{[l]}(x)$ converges almost surely towards $M_{1}$, because $\operatorname{Var}\left[f_{n}^{[l]}(x)\right] \rightarrow 0$ and one can write $f_{n}^{[l]}(x)=$ $\left[f_{n}^{[l]}(x)-E\left[f_{n}^{[l]}(x)\right]\right]+E\left[f_{n}^{[l]}(x)\right]$. 
Proof of Lemma 4.4. We use the decomposition

$$
\begin{aligned}
E\left[\widehat{F}_{n}^{[x, l]}(y)\right]= & \frac{E\left[\varphi_{n}^{[x, l]}(y)\right]}{E\left[f_{n}^{[l]}(x)\right]}-\frac{E\left\{\left[f_{n}^{[l]}(x)-E\left[f_{n}^{[l]}(x)\right]\right] \varphi_{n}^{[x, l]}(y)\right\}}{\left\{E\left[f_{n}^{[l]}(x)\right]\right\}^{2}} \\
& +\frac{E\left\{\left[f_{n}^{[l]}(x)-E\left[f_{n}^{[l]}(x)\right]\right]^{2} \widehat{F}_{n}^{[x, l]}(y)\right\}}{\left\{E\left[f_{n}^{[l]}(x)\right]\right\}^{2}}
\end{aligned}
$$

Let us start by studying

$$
\frac{E\left[\varphi_{n}^{[x, l]}(y)\right]}{E\left[f_{n}^{[l]}(x)\right]}-F^{[x]}(y)=\frac{\sum_{i=1}^{n} \frac{1}{\left[F\left(h_{i}\right)\right]^{l}} E\left\{\left[H\left(\frac{y-Y_{i}}{h_{i}}\right)-F^{[x]}(y)\right] K\left(\frac{\left\|x-X_{i}\right\|}{h_{i}}\right)\right\}}{\sum_{i=1}^{n} \frac{1}{\left[F\left(h_{i}\right)\right]^{l}} E\left[K\left(\frac{\left\|x-X_{i}\right\|}{h_{i}}\right)\right]} .
$$

Using (6.4) and the fact that

$$
\begin{aligned}
E\left[K\left(\frac{\left\|x-X_{i}\right\|}{h_{i}}\right)\right] & =\int_{0}^{h_{i}} K\left(\frac{t}{h_{i}}\right) \mathrm{dP}^{\left\|x-x_{i}\right\|}(t) \\
& =F\left(h_{i}\right)\left[K(1)-\int_{0}^{1}(K(s))^{\prime} \tau_{h_{i}}(s) d s\right],
\end{aligned}
$$

we obtain by (H1),

$$
\begin{aligned}
& \frac{E\left[\varphi_{n}^{[x, l]}(y)\right]}{E\left[f_{n}^{[l]}(x)\right]}-F^{[x]}(y) \\
& \quad=\frac{\sum_{i=1}^{n} h_{i}\left[F\left(h_{i}\right)\right]^{1-l}\left\{\varphi^{\prime}(0)\left[K(1)-\int_{0}^{1}(s K(s))^{\prime} \tau_{h_{i}}(s) d s\right]+\gamma_{i}\right\}}{\sum_{i=1}^{n}\left[F\left(h_{i}\right)\right]^{1-l}\left[K(1)-\int_{0}^{1}(K(s))^{\prime} \tau_{h_{i}}(s) d s\right]}=\frac{D_{1}}{D_{2}} .
\end{aligned}
$$

Finally, (H2), (H3) and Toeplitz' Lemma [M86] yield

$$
\frac{D_{1}}{n h_{n}\left[F\left(h_{n}\right)\right]^{1-l}}=\alpha_{[l]} \varphi^{\prime}(0) M_{0}[1+o(1)], \quad \frac{D_{2}}{n\left[F\left(h_{n}\right)\right]^{1-l}}=\beta_{[1-l]} M_{1}[1+o(1)] .
$$

And

$$
\frac{E\left[\varphi_{n}^{[x, l]}(y)\right]}{E\left[f_{n}^{[l]}(x)\right]}-F^{[x]}(y)=h_{n} \varphi^{\prime}(0) \frac{\alpha_{[l]}}{\beta_{[1-l]}} \frac{M_{0}}{M_{1}}[1+o(1)] .
$$

Now, we study the variance terms. We have

$$
\begin{aligned}
E\left[f_{n}^{[l]}(x)\right] & =\frac{1}{\sum_{i=1}^{n}\left[F\left(h_{i}\right)\right]^{1-l}} \sum_{i=1}^{n} \frac{1}{\left[F\left(h_{i}\right)\right]^{l}} E\left[K\left(\frac{\left\|x-X_{i}\right\|}{h_{i}}\right)\right] \\
& =\frac{\sum_{i=1}^{n} \frac{\left[F\left(h_{i}\right)\right]^{1-l}}{n\left[F\left(h_{n}\right)\right]^{1-l}}\left[K(1)-\int_{0}^{1}(K(s))^{\prime} \tau_{h_{i}}(s) d s\right]}{B_{n,(1-l)}} \\
& =M_{1}[1+o(1)]
\end{aligned}
$$

and 


$$
\begin{aligned}
& E\left[\varphi_{n}^{[x, l]}(y)\right]=\frac{1}{\sum_{i=1}^{n}\left[F\left(h_{i}\right)\right]^{1-l}} \sum_{i=1}^{n} \frac{1}{\left[F\left(h_{i}\right)\right]^{l}} E\left[H\left(\frac{y-Y_{i}}{h_{i}}\right) K\left(\frac{\left\|x-X_{i}\right\|}{h_{i}}\right)\right] \\
&=\frac{1}{\sum_{i=1}^{n}\left[F\left(h_{i}\right)\right]^{1-l}} \\
& \times \sum_{i=1}^{n} \frac{E\left\{\left[\int_{\mathbb{R}} H^{\prime}(t) F^{[X]}\left(y-h_{i} t\right) d t-F^{[X]}(y)+F^{[X]}(y)\right] K\left(\frac{\left\|x-X_{i}\right\|}{h_{i}}\right)\right\}}{\left[F\left(h_{i}\right)\right]^{l}} \\
&= \frac{1}{\sum_{i=1}^{n}\left[F\left(h_{i}\right)\right]^{1-l}} \sum_{i=1}^{n} \frac{1}{\left[F\left(h_{i}\right)\right]^{l}} E\left\{\left[O\left(h_{i}^{b_{2}}\right)+F^{[X]}(y)\right] K\left(\frac{\left\|x-X_{i}\right\|}{h_{i}}\right)\right\} \\
&= \frac{1}{\sum_{i=1}^{n}\left[F\left(h_{i}\right)\right]^{1-l}} \sum_{i=1}^{n} \frac{1}{\left[F\left(h_{i}\right)\right]^{l}} F\left(h_{i}\right) M_{1}\left[F^{[X]}(y)+O\left(h_{i}^{b_{2}}\right)\right] \\
&= F^{[X]}(y) M_{1}[1+o(1)] .
\end{aligned}
$$

Next, concerning variances and covariance, we have

$$
E^{2}\left[K\left(\frac{\left\|x-X_{i}\right\|}{h_{i}}\right)\right]=O\left[\left\{F\left(h_{i}\right)\right\}^{2}\right] .
$$

By (6.1) we have

$$
\operatorname{Var}\left[K\left(\frac{\left\|x-X_{i}\right\|}{h_{i}}\right)\right]=M_{2} F\left(h_{i}\right)\left[1+\gamma_{i}\right],
$$

where $\gamma_{i}=O\left[F\left(h_{i}\right)\right]$. Thus

$$
\begin{aligned}
\operatorname{Var}\left[f_{n}^{[l]}(x)\right] & =\left[\frac{1}{\sum_{i=1}^{n}\left[F\left(h_{i}\right)\right]^{1-l}}\right]^{2} \sum_{i=1}^{n}\left[\frac{1}{\left[F\left(h_{i}\right)\right]^{l}}\right]^{2} M_{2} F\left(h_{i}\right)\left[1+\gamma_{i}\right] \\
& =\frac{1}{\left(\sum_{i=1}^{n}\left[F\left(h_{i}\right)\right]^{1-l}\right)^{2}} \sum_{i=1}^{n}\left[F\left(h_{i}\right)\right]^{1-2 l} M_{2}\left[1+\gamma_{i}\right] \\
& =\frac{\beta_{[1-2 l]}}{\beta_{[1-l]}^{2}} \frac{1}{n F\left(h_{n}\right)} M_{2}[1+o(1)] .
\end{aligned}
$$

Then

$\operatorname{Var}\left[\varphi_{n}^{[x, l]}(y)\right]$

$$
=\left[\frac{1}{\sum_{i=1}^{n}\left[F\left(h_{i}\right)\right]^{1-l}}\right]^{2} \sum_{i=1}^{n}\left[\frac{1}{\left[F\left(h_{i}\right)\right]^{l}}\right]^{2} \operatorname{Var}\left[K\left(\frac{\left\|x-X_{i}\right\|}{h_{i}}\right) H\left(\frac{y-Y_{i}}{h_{i}}\right)\right],
$$

where

$$
\begin{aligned}
\operatorname{Var} & {\left[K\left(\frac{\left\|x-X_{i}\right\|}{h_{i}}\right) H\left(\frac{y-Y_{i}}{h_{i}}\right)\right] } \\
& =E\left[K\left(\frac{\left\|x-X_{i}\right\|}{h_{i}}\right) H\left(\frac{y-Y_{i}}{h_{i}}\right)\right]^{2}-E^{2}\left[K\left(\frac{\left\|x-X_{i}\right\|}{h_{i}}\right) H\left(\frac{y-Y_{i}}{h_{i}}\right)\right] .
\end{aligned}
$$


As

$$
E^{2}\left[K\left(\frac{\left\|x-X_{i}\right\|}{h_{i}}\right) H\left(\frac{y-Y_{i}}{h_{i}}\right)\right]=O\left[\left\{F\left(h_{i}\right)\right\}^{2}\right]
$$

and

$$
\begin{aligned}
& E\left[K\left(\frac{\left\|x-X_{i}\right\|}{h_{i}}\right) H\left(\frac{y-Y_{i}}{h_{i}}\right)\right]^{2} \\
& =E\left\{K^{2}\left(\frac{\left\|x-X_{i}\right\|}{h_{i}}\right) E^{2}\left[H\left(\frac{y-Y_{i}}{h_{i}}\right) \mid X\right]\right\}+E\left\{\sigma_{\varepsilon i}^{2}(X) K\left(\frac{\left\|x-X_{i}\right\|}{h_{i}}\right)\right\}
\end{aligned}
$$

with

$$
E^{2}\left[H\left(\frac{y-Y_{i}}{h_{i}}\right) \mid X\right]=O\left(h_{i}^{b_{2}}\right)+\left[F^{[X]}(y)\right]^{2},
$$

where $\sigma_{\varepsilon i}^{2}(X)$ is defined in Remark 3.1, by (H6)(ii) we have

$$
\begin{aligned}
& \operatorname{Var}\left[\varphi_{n}^{[x, l]}(y)\right] \\
& =\left[\frac{1}{\sum_{i=1}^{n}\left[F\left(h_{i}\right)\right]^{1-l}}\right]^{2} \sum_{i=1}^{n}\left[\frac{1}{\left[F\left(h_{i}\right)\right]^{l}}\right]^{2} M_{2} F\left(h_{i}\right)\left[\left[F^{[X]}(y)\right]^{2}+\sigma_{\varepsilon}^{2}(X)\right]\left[1+\gamma_{i}\right] \\
& =\frac{\beta_{[1-2 l]}}{\beta_{[1-l]}^{2}}\left[\left[F^{[X]}(y)\right]^{2}+\sigma_{\varepsilon}^{2}(X)\right] \frac{1}{n F\left(h_{n}\right)} M_{2}[1+o(1)],
\end{aligned}
$$

with $\gamma_{i}=o\left(h_{i}\right)$.

Finally,

$\operatorname{Cov}\left[f_{n}^{[l]}(x), \varphi_{n}^{[x, l]}(y)\right]$

$$
\begin{aligned}
= & \frac{1}{\left(\sum_{i=1}^{n}\left[F\left(h_{i}\right)\right]^{1-l}\right)^{2}}\left\{E\left[\sum_{i=1}^{n} \sum_{j=1}^{n} \frac{H\left(\frac{y-Y_{i}}{h_{i}}\right) K\left(\frac{\left\|x-X_{i}\right\|}{h_{i}}\right) K\left(\frac{\left\|x-X_{j}\right\|}{h_{j}}\right)}{\left[F\left(h_{i}\right)\right]^{l}\left[F\left(h_{j}\right)\right]^{l}}\right]\right. \\
& \left.-\sum_{i=1}^{n} \frac{E\left[H\left(\frac{y-Y_{i}}{h_{i}}\right) K\left(\frac{\left\|x-X_{i}\right\|}{h_{i}}\right)\right]}{\left[F\left(h_{i}\right)\right]^{l}} \sum_{j=1}^{n} \frac{E\left[K\left(\frac{\left\|x-X_{j}\right\|}{h_{j}}\right)\right]}{\left[F\left(h_{j}\right)\right]^{l}}\right\} \\
= & \frac{1}{\left(\sum_{i=1}^{n}\left[F\left(h_{i}\right)\right]^{1-l}\right)^{2}} \sum_{i=1}^{n} \frac{E\left[H\left(\frac{y-Y_{i}}{h_{i}}\right) K^{2}\left(\frac{\left\|x-X_{i}\right\|}{h_{i}}\right)\right]}{\left[F\left(h_{i}\right)\right]^{2 l}} \\
& -\frac{1}{\left(\sum_{i=1}^{n}\left[F\left(h_{i}\right)\right]^{1-l}\right)^{2}} \sum_{i=1}^{n} \frac{E\left[H\left(\frac{y-Y_{i}}{h_{i}}\right) K\left(\frac{\left\|x-X_{i}\right\|}{h_{i}}\right)\right] E\left[K\left(\frac{\left\|x-X_{i}\right\|}{h_{i}}\right)\right]}{\left[F\left(h_{i}\right)\right]^{2 l}} \\
= & I-I I,
\end{aligned}
$$

where

$$
I I=O\left[\frac{1}{n}\left(B_{n, 1-l}\right)^{-2} B_{n, 2(1-l)}\right]=O\left(\frac{1}{n F\left(h_{n}\right)}\right) .
$$


And as

$$
E\left[H\left(\frac{y-Y_{i}}{h_{i}}\right) K^{2}\left(\frac{\left\|x-X_{i}\right\|}{h_{i}}\right)\right]=F\left(h_{i}\right) M_{2}\left[F^{[X]}(y)+\gamma_{i}\right]
$$

with $\gamma_{i}=o\left(h_{i}\right)$, we have

$$
I=\frac{\left(B_{n, 1-l}\right)^{-2}}{n F\left(h_{n}\right)} \sum_{i=1}^{n} \frac{\left[F\left(h_{i}\right)\right]^{1-2 l}}{n\left[F\left(h_{n}\right)\right]^{1-2 l}} M_{2} F^{[X]}(y)\left[1+\gamma_{i}\right] .
$$

Thus

$$
\operatorname{Cov}\left[f_{n}^{[l]}(x), \varphi_{n}^{[x, l]}(y)\right]=\frac{\beta_{[1-2 l]}}{\beta_{[1-l]}^{2}} F^{[X]}(y) M_{2} \frac{1}{n F\left(h_{n}\right)}[1+o(1)] .
$$

Finally,

$$
\operatorname{Var}\left[\widehat{F}_{n}^{[x, l]}(y)\right]=\frac{\beta_{[1-2 l]}}{\beta_{[1-l]}^{2}} \frac{M_{2}}{M_{1}^{2}} \sigma_{\varepsilon}^{2}(X) \frac{1}{n F\left(h_{n}\right)}[1+o(1)] .
$$

And since

$$
\begin{aligned}
E\left\{\left[f_{n}^{[l]}(x)-E\left[f_{n}^{[l]}(x)\right]\right] \varphi_{n}^{[x, l]}(y)\right\} & =O\left[\frac{1}{n F\left(h_{n}\right)}\right], \\
E\left\{\left[f_{n}^{[l]}(x)-f(x)\right]^{2} \widehat{F}_{n}^{[x, l]}(y)\right\} & =O\left[\frac{1}{n F\left(h_{n}\right)}\right],
\end{aligned}
$$

we get

$$
E\left[\widehat{F}_{n}^{[x, l]}(y)-F^{[x]}(y)\right]=h_{n} \varphi^{\prime}(0) \frac{\alpha_{[l]}}{\beta_{[1-l]}} \frac{M_{0}}{M_{1}}[1+o(1)]+O\left[\frac{1}{n F\left(h_{n}\right)}\right] .
$$

Proof of Lemma 4.5. It is sufficient to use the following decomposition of variance that can be found in [C76]:

$$
\begin{aligned}
\operatorname{Var}\left[\widehat{F}_{n}^{[x, l]}(y)\right]= & \frac{\operatorname{Var}\left[\varphi_{n}^{[x, l]}(y)\right]}{\left\{E\left[f_{n}^{[l]}(x)\right]\right\}^{2}}-4 \frac{E\left[\varphi_{n}^{[x, l]}(y)\right] \operatorname{Cov}\left[f_{n}^{[l]}(x), \varphi_{n}^{[x, l]}(y)\right]}{\left\{E\left[f_{n}^{[l]}(x)\right]\right\}^{3}} \\
& +3 \operatorname{Var}\left[f_{n}^{[l]}(x)\right] \frac{\left\{E\left[\varphi_{n}^{[x, l]}(y)\right]\right\}^{2}}{\left\{E\left[f_{n}^{[l]}(x)\right]\right\}^{4}}+o\left[\frac{1}{n F\left(h_{n}\right)}\right],
\end{aligned}
$$

and the same steps used in the proof of Lemma 4.5.

Proof of Theorem 4.3. The result is a direct consequence of Lemmas 4.4 and 4.5 .

Proof of Theorem 4.7. Let

$$
\widehat{f}_{n}^{[x, l]}(y)-f^{[x]}(y)=\frac{\phi_{n}^{[x, l]}(y)-f^{[x]}(y) f_{n}^{[l]}(x)}{f_{n}^{[l]}(x)},
$$


where

$$
\begin{aligned}
& \varphi_{n}^{[x, l]}(y)-f^{[x]}(y) f_{n}^{[l]}(x) \\
&=\left\{\varphi_{n}^{[x, l]}(y)-f^{[x]}(y) f_{n}^{[l]}(y)-E\left[\varphi_{n}^{[x, l]}(y)-f^{[x]}(y) f_{n}^{[l]}(x)\right]\right\} \\
&+\left\{E\left[\varphi_{n}^{[x, l]}(y)-f^{[x]}(y) f_{n}^{[l]}(x)\right]\right\} \\
&= I_{1}^{\prime}+I_{2}^{\prime} .
\end{aligned}
$$

The proofs for $I_{1}^{\prime}$ and $I_{2}^{\prime}$ are similar to those for $I_{1}$ and $I_{2}$, and we find

$$
\begin{aligned}
\varlimsup_{n \rightarrow \infty}\left\{\frac{n F\left(h_{n}\right)}{\ln \ln n}\right\}^{1 / 2} I_{1}^{\prime} & =\frac{\left\{2 \varpi_{[1-2 l]} M_{2} f^{[x]}(y) \int_{\mathbb{R}} H^{\prime 2}(t) d t\right\}^{1 / 2}}{\beta_{[1-l]}}, \\
I_{2}^{\prime} & \simeq h_{n} \phi^{\prime}(0) \frac{\alpha_{[l]}}{\beta_{[1-l]}} M_{0}[1+o(1)] .
\end{aligned}
$$

Then

$$
\left\{\frac{n F\left(h_{n}\right)}{\ln \ln n}\right\}^{1 / 2} I_{2}^{\prime}=\left\{\frac{n F\left(h_{n}\right)}{\ln \ln n}\right\}^{1 / 2} h_{n} \phi^{\prime}(0) \frac{\alpha_{[l]}}{\beta_{[1-l]}} M_{0}[1+o(1)]=o(1)
$$

when $\lim _{n \rightarrow \infty} n h_{n}^{2}=0$. We conclude that

$$
\varlimsup_{n \rightarrow \infty}\left\{\frac{n F\left(h_{n}\right)}{\ln \ln n}\right\}^{1 / 2} I_{2}^{\prime}=0
$$

and

$$
\begin{aligned}
\left\{\frac{n F\left(h_{n}\right)}{\ln \ln n}\right\}^{1 / 2}\left[\varphi_{n}^{[x, l]}(y)-f^{[x]}(y) f_{n}^{[l]}(x)\right] & \\
& \rightarrow \frac{\left\{2 \varpi_{[1-2 l]} M_{2} f^{[x]}(y) \int_{\mathbb{R}} H^{\prime 2}(t) d t\right\}^{1 / 2}}{\beta_{[1-l]}} .
\end{aligned}
$$

Proof of Lemma 4.9. Following the same reasoning for the conditional cumulative distribution function, we have

$$
\begin{aligned}
E\left[f_{n}^{[l]}(x)\right] & =M_{1}[1+o(1)] ; E\left[\varphi_{n}^{[x, l]}(y)\right]=f^{[X]}(y) M_{1}[1+o(1)], \\
\operatorname{Var}\left[f_{n}^{[l]}(x)\right] & =\frac{\beta_{[1-2 l]}}{\beta_{[1-l]}^{2}} \frac{1}{n F\left(h_{n}\right)} M_{2}[1+o(1)], \\
\operatorname{Var}\left[\varphi_{n}^{[x, l]}(y)\right] & =\frac{\beta_{[1-2 l]}}{\beta_{[1-l]}^{2}}\left[\left[f^{[X]}(y)\right]^{2}+\theta_{\varepsilon}^{2}(X)\right] \frac{1}{n F\left(h_{n}\right)} M_{2}[1+o(1)]
\end{aligned}
$$

$\left(\theta_{\varepsilon}^{2}(X)\right.$ is defined in Remark 3.1). Moreover

$$
\operatorname{Cov}\left[f_{n}^{[l]}(x), \varphi_{n}^{[x, l]}(y)\right]=\frac{\beta_{[1-2 l]}}{\beta_{[1-l]}^{2}} f^{[X]}(y) M_{2} \frac{1}{n F\left(h_{n}\right)}[1+o(1)] .
$$


Replacing the parameters by their respective values, one finds

$$
\operatorname{Var}\left[\widehat{f}_{n}^{[x, l]}(y)\right]=\frac{\beta_{[1-2 l]}}{\beta_{[1-l]}^{2}} \frac{M_{2}}{M_{1}^{2}} \theta_{\varepsilon}^{2}(X) \frac{1}{n F\left(h_{n}\right)}[1+o(1)] .
$$

Then, given

and

$$
E\left\{\left[f_{n}^{[l]}(x)-E f_{n}^{[l]}(x)\right] \varphi_{n}^{[x, l]}(y)\right\}=O\left[\frac{1}{n F\left(h_{n}\right)}\right]
$$

$$
E\left\{\left[f_{n}^{[l]}(x)-f(x)\right]^{2} \widehat{f}_{n}^{[x, l]}(y)\right\}=O\left[\frac{1}{n F\left(h_{n}\right)}\right]
$$

we have

$$
E\left[\widehat{f}_{n}^{[x, l]}(y)-f^{[x]}(y)\right]=h_{n} \phi^{\prime}(0) \frac{\alpha_{[l]}}{\beta_{[1-l]}} \frac{M_{0}}{M_{1}}[1+o(1)]+O\left[\frac{1}{n F\left(h_{n}\right)}\right] .
$$

Proof of Lemma 4.10. It is sufficient to look at (6.7).

Proof of Theorem 4.8. This is a direct consequence of Lemmas 4.9 and 4.10.

Proof of Theorem 4.12. Consider the decomposition

$$
\begin{aligned}
\limsup _{n \rightarrow \infty}\left[\frac{n F\left(h_{n}\right)}{\ln \ln n}\right]^{1 / 2}\left[\widehat{h}_{n}^{[x, l]}(y)-h^{[x]}(y)\right] \\
=\limsup _{n \rightarrow \infty}\left[\frac{n F\left(h_{n}\right)}{\ln \ln n}\right]^{1 / 2} \frac{1}{1-\widehat{F}_{n}^{[x, l]}(y)}\left(\widehat{f}_{n}^{[x, l]}(y)-f^{[x]}(y)\right) \\
\quad+h^{[x]}(y) \limsup _{n \rightarrow \infty}\left[\frac{n F\left(h_{n}\right)}{\ln \ln n}\right]^{1 / 2} \frac{1}{1-\widehat{F}_{n}^{[x, l]}(y)}\left(\widehat{F}_{n}^{[x, l]}(y)-F^{[x]}(y)\right) .
\end{aligned}
$$

Via 6.5 and 6.6), we get the almost sure convergence of $\widehat{F}_{n}^{[x, l]}(y)$ to $F^{[x]}(y)$. Then the convergence of the denominator $1-\widehat{F}_{n}^{[x, l]}(y)$ to $1-F^{[x]}(y)$ is obtained almost surely. The proof is completed by using Theorems 4.1 and 4.7 .

Proof of Theorem 4.13. Using the decomposition of $\widehat{h}_{n}^{[x, l]}(y)$ deduced from [BC99], we have

$$
\begin{aligned}
E\left[\widehat{h}_{n}^{[x, l]}(y)-\right. & \left.h^{[x]}(y)\right]^{2} \\
= & \frac{\left[h^{[x]}(y)\right]^{2}}{\left[1-F^{[x]}(y)\right]^{2}} E\left[\widehat{F}_{n}^{[x, l]}(y)-F^{[x]}(y)\right]^{2} \\
& +\frac{2 h^{[x]}(y)}{\left[1-F^{[x]}(y)\right]^{2}} E\left\{\left[\widehat{F}_{n}^{[x, l]}(y)-F^{[x]}(y)\right]\left[\widehat{f}_{n}^{[x, l]}(y)-f^{[x]}(y)\right]\right\} \\
& +\frac{1}{\left[1-F^{[x]}(y)\right]^{2}} E\left[\widehat{f}_{n}^{[x, l]}(y)-f^{[x]}(y)\right]^{2}
\end{aligned}
$$




$$
\begin{aligned}
& +\frac{1}{\left[1-F^{[x]}(y)\right]^{2}} E\left\{\left[\left(\widehat{h}_{n}^{[x, l]}(y)\right)^{2}-\left(h^{[x]}(y)\right)^{2}\right]\left[\widehat{F}_{n}^{[x, l]}(y)-F^{[x]}(y)\right]^{2}\right\} \\
& +\frac{2}{\left[1-F^{[x]}(y)\right]^{2}} \\
& \quad \times E\left\{\left[\widehat{h}_{n}^{[x, l]}(y)-h^{[x]}(y)\right]\left[\widehat{f}_{n}^{[x, l]}(y)-f^{[x]}(y)\right]\left[\widehat{F}_{n}^{[x, l]}(y)-F^{[x]}(y)\right]\right\} \\
& =\Lambda_{1}+\Lambda_{2}+\Lambda_{3}+\Lambda_{4}+\Lambda_{5} .
\end{aligned}
$$

Thus, the quadratic mean convergence of $\widehat{h}_{n}^{[x, l]}(y)$ into $h^{[x]}(y)$ depends on the convergence of $\Lambda_{1}, \Lambda_{2}, \Lambda_{3}, \Lambda_{4}$ and $\Lambda_{5}$.

Studying $\Lambda_{1}$. As

$$
\Lambda_{1}=\frac{\left[h^{[x]}(y)\right]^{2}}{\left[1-F^{[x]}(y)\right]^{2}} E\left[\widehat{F}_{n}^{[x, l]}(y)-F^{[x]}(y)\right]^{2},
$$

using the quadratic mean convergence of $\widehat{F}_{n}^{[x, l]}(y)$ to $F^{[x]}(y)$ we obtain

$$
\lim _{n \rightarrow \infty} n F\left(h_{n}\right) \Lambda_{1}=\frac{\left[h^{[x]}(y)\right]^{2}}{\left[1-F^{[x]}(y)\right]^{2}}\left\{\frac{\beta_{[1-2 l]}}{\beta_{[1-l]}^{2}} \frac{M_{2}}{M_{1}^{2}} \sigma_{\varepsilon}^{2}(X)+c\left[\varphi^{\prime}(0)\right]^{2} \frac{\alpha_{[l]}^{2}}{\beta_{[1-l]}^{2}} \frac{M_{0}^{2}}{M_{1}^{2}}\right\} .
$$

Studying $\Lambda_{2}$. We have

$$
\Lambda_{2}=\frac{2 h^{[x]}(y)}{\left[1-F^{[x]}(y)\right]^{2}} E\left\{\left[\widehat{F}_{n}^{[x, l]}(y)-F^{[x]}(y)\right]\left[\widehat{f}_{n}^{[x, l]}(y)-f^{[x]}(y)\right]\right\}
$$

and

$$
\begin{aligned}
E\left\{\left[\widehat{F}_{n}^{[x, l]}(y)-F^{[x]}(y)\right]\left[\widehat{f}_{n}^{[x, l]}(y)-f^{[x]}(y)\right]\right\} & \\
& =\left[E \widehat{f}_{n}^{[x, l]}(y)-f^{[x]}(y)\right]\left[E \widehat{F}_{n}^{[x, l]}(y)-F^{[x]}(y)\right]+\operatorname{Cov}\left[\widehat{f}_{n}^{[x, l]}(y), \widehat{F}_{n}^{[x, l]}(y)\right] .
\end{aligned}
$$

By using 6.6 and (6.8), suppose there exists $c>0$ such that $n F\left(h_{n}\right) h_{n}^{2} \rightarrow c$ as $n \rightarrow \infty$, then

$\lim _{n \rightarrow \infty} n F\left(h_{n}\right)\left[E \widehat{f}_{n}^{[x, l]}(y)-f^{[x]}(y)\right]\left[E \widehat{F}_{n}^{[x, l]}(y)-F^{[x]}(y)\right]=c \phi^{\prime}(0) \varphi^{\prime}(0) \frac{\alpha_{[l]}^{2}}{\beta_{[1-l]}^{2}} \frac{M_{0}^{2}}{M_{1}^{2}}$.

We can prove that $n F\left(h_{n}\right) \operatorname{Cov}\left[\widehat{f}_{n}^{[x, l]}(y), \widehat{F}_{n}^{[x, l]}(y)\right] \rightarrow 0$.

Effectively, by the Schwarz inequality,

$$
\operatorname{Cov}\left[\widehat{f}_{n}^{[x, l]}(y), \widehat{F}_{n}^{[x, l]}(y)\right] \leq \sqrt{\operatorname{Var}\left(\widehat{f}_{n}^{[x, l]}(y)\right) \operatorname{Var}\left(\widehat{F}_{n}^{[x, l]}(y)\right)}
$$

Using 6.6 and (6.7), we find

$$
\begin{aligned}
\operatorname{Cov}\left[\widehat{f}_{n}^{[x, l]}(y), \widehat{F}_{n}^{[x, l]}(y)\right] & \\
& \simeq \frac{\beta_{[1-2 l]}}{\beta_{[1-l]}^{2}} \frac{M_{2}}{M_{1}^{2}} \sqrt{\theta_{\varepsilon}^{2}(X) \sigma_{\varepsilon}^{2}(X)} \frac{1}{n F\left(h_{n}\right)}[1+O(1)] \quad \text { as } n \rightarrow \infty
\end{aligned}
$$


We then get

$$
\begin{aligned}
\lim _{n \rightarrow \infty} n F\left(h_{n}\right) \Lambda_{2} \\
\quad=\frac{2 h^{[x]}(y)}{\left[1-F^{[x]}(y)\right]^{2}}\left[c \phi^{\prime}(0) \varphi^{\prime}(0) \frac{\alpha_{[l]}^{2}}{\beta_{[1-l]}^{2}} \frac{M_{0}^{2}}{M_{1}^{2}}+\frac{\beta_{[1-2 l]}}{\beta_{[1-l]}^{2}} \frac{M_{2}}{M_{1}^{2}} \sqrt{\theta_{\varepsilon}^{2}(X) \sigma_{\varepsilon}^{2}(X)}\right] .
\end{aligned}
$$

Studying $\Lambda_{3}$. Recall

$$
\Lambda_{3}=\frac{1}{\left[1-F^{[x]}(y)\right]^{2}} E\left[\widehat{f}_{n}^{[x, l]}(y)-f^{[x]}(y)\right]^{2} .
$$

By using the result of Theorem 4.8 we find

$$
\lim _{n \rightarrow \infty} n F\left(h_{n}\right) \Lambda_{3}=\frac{1}{\left[1-F^{[x]}(y)\right]^{2}}\left[\frac{\beta_{[1-2 l]}}{\beta_{[1-l]}^{2}} \frac{M_{2}}{M_{1}^{2}} \theta_{\varepsilon}^{2}(X)+c\left[\phi^{\prime}(0)\right]^{2} \frac{\alpha_{[l]}^{2}}{\beta_{[1-l]}^{2}} \frac{M_{0}^{2}}{M_{1}^{2}}\right] \text {. }
$$

Studying $\Lambda_{4}$. We have

$$
\Lambda_{4}=\frac{1}{\left[1-F^{[x]}(y)\right]^{2}} E\left\{\left[\left(\widehat{h}_{n}^{[x, l]}(y)\right)^{2}-\left(h^{[x]}(y)\right)^{2}\right]\left[\widehat{F}_{n}^{[x, l]}(y)-F^{[x]}(y)\right]^{2}\right\} .
$$

Since $\left(\widehat{h}_{n}^{[x, l]}(y)\right)^{2}-\left(h^{[x]}(y)\right)^{2}$ can be bounded by a constant, the convergence of $\Lambda_{4}$ can be deduced easily using the quadratic mean convergence of $\widehat{F}_{n}^{[x, l]}(y)$ to $F^{[x]}(y)$. For this, we use the almost sure convergence of $\widehat{h}_{n}^{[x, l]}(y)$ to $h^{[x]}(y)$. Hence

$$
\lim _{n \rightarrow \infty} n F\left(h_{n}\right) \Lambda_{4}=0 .
$$

Studying $\Lambda_{5}$. Using the same procedure as for $\Lambda_{4}$, we get

$$
\lim _{n \rightarrow \infty} n F\left(h_{n}\right) \Lambda_{5}=0
$$

7. Conclusion and future scope. In this work, our main interest was nonparametric estimation methods based on conditional recursive kernels for independent functional data along with their applications in prediction. We focused on recursive estimation of the conditional hazard function; to this end, new nonparametric models and estimates were determined, that is, recursive estimators of the conditional cumulative distribution and the density functions. Then, taking the recursive estimation approach, we established the almost sure and the quadratic mean convergence rates of the proposed estimators. In addition, we analyzed the performance of our estimator using a simulation study; our prime purpose was to improve the performance of nonparametric conditional kernel predictors, reducing their computation time using recursive kernels. Here, our proposed estimator showed a significant advantage as regards computation time compared to the classical nonparametric (nonrecursive) functional estimator given in [BRS15]. Further, in order to explore the effectiveness of our technique in real cases, we 
presented the convenient use of our model to study a time series, specifically, the El Niño time series. Here, the nonrecursive estimator showed a slight advantage over our estimator with regard to the mean prediction error, but there is not a big difference between their behaviors, while our recursive estimator has better computation time. For further work, it will be interesting to extend the present study by examining the model under strong mixing conditions.

Acknowledgements. The authors are grateful to the anonymous referees and the editor for insightful comments and suggestions, which improved the content and the presentation of this paper.

\section{References}

[A02] C. Acerbi, Spectral measures of risk: A coherent representation of subjective risk aversion, J. Banking Finance 26 (2002), 1505-1518.

[ACT14] A. Amiri, C. Crambes and B. Thiam, Recursive estimation of nonparametric regression with functional covariate, Comput. Statist. Data Anal. 69 (2014), $154-172$.

[AT16] A. Amiri and B. Thiam, Regression estimation by local polynomial fitting for multivariate data streams, Statist. Papers 59 (2018), 813-843.

[ABCV17] G. Aneiros et al. (eds.), Functional Statistics and Related Fields, Springer, 2017.

[BL94] B. H. Baltagi and Q. Li, A simple recursive estimation method for linear regression models with $\mathrm{AR}(p)$ disturbances, Statist. Papers 35 (1994), 93-100.

[BRS15] N. Belkhir, A. Rabhi and S. Soltani, Exact asymptotic errors of the hazard conditional rate kernel, J. Statist. Appl. Probab. Lett. 2 (2015), 191-204.

[BFRV07] K. Benhenni, F. Ferraty, M. Rachdi and P. Vieu, Local smoothing regression with functional data, Comput. Statist. 22 (2007), 353-369.

[BC99] D. Bosq and N. Cheze-Payaud, Optimal asymptotic quadratic error of nonparametric regression function estimates for a continuous-time process from sampled-data, Statistics 32 (1999), 229-247.

[B14] A. Bouadjemi, Asymptotic normality of the recursive kernel estimate of conditional cumulative distribution function, J. Probab. Statist. Sci. 12 (2014), $117-126$.

[C08] Z. Cai and X. Wang, Nonparametric estimation of conditional VaR and expected shortfall, J. Econometrics 147 (2008), 120-130.

[C76] G. Collomb, Estimation nonparamétrique de la régression par la méthode du noyau, Ph.D. Thesis, Univ. Paul Sabatier, Toulouse, 1976.

[C14] A. Cuevas, A partial overview of the theory of statistics with functional data, J. Statist. Planning Inference 147 (2014), 1-23.

[D08] A. Quintela-del-Río, Hazard function given a functional variable: Non-parametric estimation under strong mixing conditions, J. Nonparametric Statist. 20 (2008), 413-430.

[EKM10] P. Embrechts, C. Klüppelberg and T. Mikosch, Modelling Extremal Events for Insurance and Finance, Springer, 2010. 
[F11] F. Ferraty (ed.), Recent Advances in Functional Data Analysis and Related Topics, Physica-Verlag, 2011.

[FMV07] F. Ferraty, A. Mas and P. Vieu, Nonparametric regression on functional data: inference and practical aspects, Austral. New Zealand J. Statist. 49 (2007), $267-286$.

[FRV08] F. Ferraty, A. Rabhi et P. Vieu, Estimation non-paramétrique de la fonction de hasard avec variable explicative fonctionnelle, Rev. Roumaine Math. Pures Appl. 53 (2008), 1-18.

[GV16] A. Goia and P. Vieu, An introduction to recent advances in high/infinite dimensional statistics, J. Multivariate Anal. 146 (2016), 1-6.

[GKWW18] T. Górecki, M. Krzyśko, L. Waszak and W. Wołyński, Selected statistical methods of data analysis for multivariate functional data, Statist. Papers 59 (2018), 153-182.

[LM14] A. Laksaci and B. Mechab, Conditional hazard estimate for functional random fields, J. Statist. Theory Practice 8 (2014), 192-220.

[M86] E. Masry, Recursive probability density estimation for weakly dependent stationary processes, IEEE Trans. Information Theory 32 (1986), 254-267.

[R92] G. G. Roussas, Exact rates of almost sure convergence of a recursive kernel estimate of a probability densiy function: Application to regression and hazard rate estimation, J. Nonparametric Statist. 1 (1992), 171-195.

[S04] O. Scaillet, Nonparametric estimation and sensitivity analysis of expected shortfall, Math. Finance 14 (2004), 115-129.

[T11] A. Tadj, Sur les modèles non paramétriques conditionnels en statistique fonctionnelle, thèse, Univ. Toulouse 3 Paul Sabatier, 2011.

[WW69] C. Wolverton and T. Wagner, Recursive estimates of probability densities, IEEE Trans. Systems Sci. Cybernetics 5 (1969), 246-247.

Amina Angelika Bouchentouf, Abbes Rabhi

Laboratory of Mathematics

University Djillali Liabes of Sidi Bel Abbes

Sidi Bel Abbes, Algeria

E-mail: bouchentouf_amina@yahoo.fr rabhi_abbes@yahoo.fr
Aboubacar Traore Faculty of Sciences and Techniques of Bamako Bamako, Mali E-mail: ababu02@yahoo.fr 
